\title{
PING-PONG AND OUTER SPACE
}

\author{
ILYA KAPOVICH AND MARTIN LUSTIG
}

\begin{abstract}
We prove that, if $\varphi, \psi \in \operatorname{Out}\left(F_{N}\right)$ are hyperbolic iwips (irreducible with irreducible powers) such that $\langle\varphi, \psi\rangle \subseteq \operatorname{Out}\left(F_{N}\right)$ is not virtually cyclic, then some high powers of $\varphi$ and $\psi$ generate a free subgroup of rank two for which all non-trivial elements are again hyperbolic iwips. Being a hyperbolic iwip element of $\operatorname{Out}\left(F_{N}\right)$ is strongly analogous to being a pseudo-Anosov element of a mapping class group, so the above result provides analogues of "purely pseudo-Anosov" free subgroups in $\operatorname{Out}\left(F_{N}\right)$.
\end{abstract}

\section{Contents}

1. Introduction

2. Outer space and the space of geodesic currents 6

2.1. Outer space 6

2.2. Geodesic currents 7

2.3. Intersection form 8

3. Stabilizers of eigentrees and eigencurrents 8

4. North-South Dynamics, standard neighborhoods and height functions

5. Ping-pong for Schottky type groups and its consequences 19

6. Every Schottky group contains a rank-two free iwip subgroup 24

References

\section{INTRODUCTION}

One of the most important sources for understanding free group automorphisms is the analogy with surface groups and mapping classes. Many key concepts from Thurston's approach to Teichmüller theory have been successfully carried over to the $\operatorname{Out}\left(F_{N}\right)$ world, most notably Culler-Vogtmann's Outer space [18], and Bestvina-Handel's train track representatives [3]. However, often the situation for $\operatorname{Out}\left(F_{N}\right)$ turns out to be more difficult (but also much richer in interesting phenomena) than in the parallel mapping class cosmos. One such fundamental situation arises with the translation of the concept of pseudo-Anosov homeomorphisms to free group automorphisms.

2000 Mathematics Subject Classification. Primary 20F, Secondary 57M, 37B, 37D.

The first author was supported by the NSF grants DMS-0603921 and DMS-0904200. 
It turns out that there are two such possible translations, both natural and interesting:

The first translation is based on the characterization of pseudo-Anosov homeomorphisms $h: \Sigma \rightarrow \Sigma$ (where $\Sigma$ is a closed hyperbolic surface) as precisely those which give a mapping torus $\Sigma \rtimes_{h} \mathbb{S}^{1}$ that admits a hyperbolic structure.

This is equivalent to the condition that $\pi_{1}\left(\Sigma \rtimes_{h} \mathbb{S}^{1}\right)$ is Gromov-hyperbolic. By analogy, one can consider hyperbolic automorphisms $\Phi: F_{N} \rightarrow F_{N}$, defined by the fact that the mapping torus group $G_{\Phi}=F_{N} \rtimes_{\Phi} \mathbb{Z}$ is wordhyperbolic. The Bestvina-Feighn Combination Theorem [1] implies that an automorphism $\Phi$ of $F_{N}$ is hyperbolic if and only if for some (and hence any) free basis $A$ of $F_{N}$ there exist $M \geq 1$ and $\lambda>1$ such that for every $w \in F_{N}$ we have $\lambda|w|_{A} \leq \max \left\{\left|\Phi^{M}(w)\right|_{A},\left|\Phi^{-M}(w)\right|_{A}\right\}$. This latter condition is often taken as the definition of an automorphism $\Phi$ of $F_{N}$ for being hyperbolic. It is not hard to see that whether $\Phi \in \operatorname{Aut}\left(F_{N}\right)$ is hyperbolic or not depends only on the outer automorphism class $\varphi$ of $\Phi$ in $\operatorname{Out}\left(F_{N}\right)$. An important result of Brinkmann 12 shows that $\varphi \in \operatorname{Out}\left(F_{N}\right)$ is hyperbolic if and only if $\varphi$ is atoroidal, that is, if there does not exist a non-trivial conjugacy class in $F_{N}$ that is fixed by some positive power of $\varphi$.

The second translation of the notion of being pseudo-Anosov to the free group setting is based on the dynamical properties of pseudo-Anosov homeomorphisms: a homeomorphism $h: \Sigma \rightarrow \Sigma$ is pseudo-Anosov if and only if $h$ and hence any positive power of $h$ is not reducible. However, in the free group setting the notion of reducible automorphisms $\Phi: F_{N} \rightarrow F_{N}$ is much more delicate than for surfaces: If $h$ fixes (up to isotopy) an essential subsurface of $\Sigma$, than it also fixes the complementary subsurface. But is is easy to find examples where $\Phi$ fixes a proper free factor of $F_{N}$, but no complementary free factor is is mapped to a conjugate of itself.

In this context, the notion of being irreducible for elements of $\operatorname{Out}\left(F_{N}\right)$ (see Definition 3.1 below) has been proposed in [3, but contrary to "pseudoAnosov", the property "irreducible" is not stable under replacing the automorphism by a positive power. More useful seems the following notion: An element $\varphi \in \operatorname{Out}\left(F_{N}\right)$ (or any of its lifts $\Phi \in \operatorname{Aut}\left(F_{N}\right)$ ) is said to be $i r$ reducible with irreducible powers or an iwip for short, if for every $n \geq 1$ the power $\varphi^{n}$ is irreducible (sometimes such automorphisms are also called fully irreducible). It is not hard to see that $\varphi \in \operatorname{Out}\left(F_{N}\right)$ is an iwip if and only if no positive power of $\varphi$ preserves the conjugacy class of a proper free factor of $F_{N}$ (and one can take the latter condition as the definition of being an iwip). It is easy to construct examples of elements of $\operatorname{Out}\left(F_{N}\right)$ that are hyperbolic but reducible. Similarly, there exists non-hyperbolic iwips (they come from pseudo-Anosov homeomorphisms of once-punctured surfaces). Thus the notions of being iwip and being hyperbolic are logically independent.

Both of these free group analogues of being pseudo-Anosov play an important role in the study of $\operatorname{Out}\left(F_{N}\right)$. Iwips have nicer properties: for example, 
they act with "North-South" dynamics on the Thurston compactification of Outer space [41] (just as pseudo-Anosovs do on Teichmüller space and its Thurston boundary). Hyperbolic automorphisms, on the other hand, are easier to come by. For example, it has been shown by Bestvina, Feighn and Handel [5] that every subgroup of $\operatorname{Out}\left(F_{N}\right)$, which contains a hyperbolic iwip and which is not virtually cyclic, contains a free subgroup of rank two where every non-trivial element is hyperbolic.

The main result of this paper is the analogous statement of this last result for hyperbolic iwips (c.f. Theorem 6.2 below):

Theorem 1.1. Let $N \geq 3$ and let $\varphi, \psi \in \operatorname{Out}\left(F_{N}\right)$ be hyperbolic iwips such that the subgroup $\langle\varphi, \psi\rangle \subseteq \operatorname{Out}\left(F_{N}\right)$ is not virtually cyclic. Then there exists $m, n \geq 1$ such that the subgroup $G=\left\langle\varphi^{m}, \psi^{n}\right\rangle \subseteq \operatorname{Out}\left(F_{N}\right)$ is free of rank two and such that every nontrivial element of $G$ is again a hyperbolic iwip.

Thus the group $G$ in Theorem 1.1 is "purely hyperbolic iwip". It was already known by the results of [5] that one can ensure for every nontrivial element of $G$ as in Theorem 1.1 to be a hyperbolic automorphism, and the new result here is the iwip property. Nevertheless, we also provide a complete and independent proof of the "purely hyperbolic" property as well.

By Corollary 3.11 for two hyperbolic iwips $\varphi, \psi \in \operatorname{Out}\left(F_{N}\right)$ the condition that they don't generate a virtually cyclic subgroup is equivalent to the condition that they don't have any common non-trivial powers, that is $\langle\varphi\rangle \cap$ $\langle\psi\rangle=\{1\}$.

As a consequence of Theorem 1.1, we obtain (c.f. Corollary 6.3 below):

Corollary 1.2. Let $G \subseteq \operatorname{Out}\left(F_{N}\right)$ be a non-virtually-cyclic subgroup that contains a hyperbolic iwip. Then $G$ contains a non-abelian free subgroup where all non-trivial elements are hyperbolic iwips.

Note that results similar to the statement of Theorem 1.1 play an important role in the study of mapping class groups. Namely, for the mapping class group $\operatorname{Mod}(\Sigma)$ of a closed hyperbolic surface $\Sigma$ it is interesting to find purely pseudo-Anosov subgroups of $\operatorname{Mod}(\Sigma)$, i.e. subgroups where all nontrivial elements are pseudo-Anosov. One of the motivations in looking for purely pseudo-Anosov subgroups of $\operatorname{Mod}(\Sigma)$ is in trying to find new examples of word-hyperbolic extensions of $\pi_{1}(\Sigma)$ by groups other than infinite cyclic ones. An important early example of a non-abelian free purely pseudoAnosov subgroup $\operatorname{Mod}(\Sigma)$ is due to Mosher [46] who used it to construct a word-hyperbolic extension of $\pi_{1}(\Sigma)$ by the free group $F_{2}$. Mosher's example was based on exploiting ping-pong considerations for the action of Schottkytype subgroups of $\operatorname{Mod}(\Sigma)$ on the boundary of the Teichmuller space; these types of subgroups are basic examples of convex-cocompact subgroups of mapping class groups. Another important source of purely pseudo-Anosov subgroups of mapping class groups comes from the work of Whittlesey [49]. This topic plays a key role in the theory of convex-cocompact subgroups 
of mapping class groups [19, 24, 39, 40, and it is known that every such convex-cocompact subgroup is purely pseudo-Anosov.

A recent result of Handel and Mosher [27] characterizes those subgroups of $\operatorname{Out}\left(F_{N}\right)$ that do not contain an iwip and shows that such subgroups have a rather special structure: if a subgroup $G \subseteq \operatorname{Out}\left(F_{N}\right)$ does not contain an iwip then there is a subgroup of finite index $H \subseteq G$ such that $H$ preserves the conjugacy class of a proper free factor of $F_{N}$.

Theorem 1.1 can also be derived from a recent result of Bestvina and Feighn [8] about the existence of a hyperbolic graph with an $\operatorname{Out}\left(F_{N}\right)$-action, given a finite collection of independent hyperbolic iwips. Our proof is based on rather different and more direct arguments and we believe that it has substantial independent value, especially in view of the goal of developing the theory of convex-cocompactness for subgroups of $O u t\left(F_{N}\right)$. Note also, that a new paper of Clay and Pettet [13] provides a proof of a related statement to our Theorem 1.1: they prove that given two "sufficiently transverse" Dehn twists $\varphi, \psi \in \operatorname{Out}\left(F_{N}\right)$, for some sufficiently large $m, n \geq 1$ the subgroup $\left\langle\varphi^{m}, \psi^{n}\right\rangle \leq \operatorname{Out}\left(F_{N}\right)$ is free of rank two and every nontrivial element of that subgroup, except those that are conjugate to powers of $\varphi^{m}, \psi^{n}$, is a hyperbolic iwip. This result of Clay and Pettet and our Theorem 1.1 are logically independent and the proofs are very different. Theorem 1.1 was applied and pushed further in a new paper of Hamenstädt [26].

We establish Theorem 1.1 via studying the dynamics of the action of $\operatorname{Out}\left(F_{N}\right)$ and of its subgroups on the space $\overline{\mathrm{cv}}_{N}$ of very small isometric $\mathbb{R}$ tree actions of $F_{N}$ (which is the closure of the Outer space $\mathrm{cv}_{N}$ in the length function topology) and on the space $\operatorname{Curr}\left(F_{N}\right)$ of geodesic currents on $F_{N}$. A geodesic current is a measure-theoretic analogue of the notion of a conjugacy class in a free group (or a free homotopy class of a closed curve on the surface). Geodesic currents in the context of hyperbolic surfaces were introduced by Bonahon who used them to study the geometry of the Teichmüller space [9, 10]. In the context of free groups geodesic currents were first introduced in the Ph.D.-thesis of Reiner Martin [44] and later re-introduced and studied systematically by Kapovich [30, 31, 32], Kapovich-Lustig [33, 34, 35] and others [20, 38. Recent applications of geodesic currents include results related to free group analogues of the curve complex (Kapovich-Lustig [34, Bestvina-Feighn [8]) and to bounded cohomology of $\operatorname{Out}\left(F_{N}\right)$ and of its subgroups (Hamenstadt [25]). A key component in these results, as well as in the proofs of the main results of the present paper, is the geometric intersection form. The latter pairs very small $F_{N}$-trees and geodesic currents and shares important features in common with Bonahon's notion of a geometric intersection number between two geodesic currents. This intersection form was initially constructed in 31, 43, for the ordinary unprojectivized Outer space $\mathrm{cv}_{N}$ and recently extended in our joint paper 34 to the closure $\overline{\mathrm{cv}}_{N}$ of $\mathrm{cv}_{N}$. 
We start by exploiting the fact that a hyperbolic iwip $\varphi \in \operatorname{Out}\left(F_{N}\right)$ acts with a "North-South" dynamics on both the projectivization $\overline{\mathrm{CV}}_{N}$ of $\overline{\mathrm{cv}}_{N}$ and on the projectivization $\mathbb{P} \operatorname{Curr}\left(F_{N}\right)$ of $\operatorname{Curr}\left(F_{N}\right)$. In the process we introduce, using the intersection form, natural "height functions" associated to $\varphi$ on each of $\overline{\mathrm{CV}}_{N}$ and $\mathbb{P} \operatorname{Curr}\left(F_{N}\right)$, which provide useful stratifications of these spaces. As a corollary of the "North-South" dynamics for the action of hyperbolic iwips on $\mathbb{P C u r r}\left(F_{N}\right)$, we obtain a new proof (Theorem 4.17) of the following result of Bestvina, Feighn and Handel [5]: If $\varphi, \psi \in \operatorname{Out}\left(F_{N}\right)$ are as in Theorem 1.1 and $\Phi, \Psi \in \operatorname{Aut}\left(F_{N}\right)$ are their representatives in $\operatorname{Aut}\left(F_{N}\right)$, then for sufficiently high powers $\Phi^{n}, \Psi^{m}$ of $\Phi$ and $\Psi$, the semidirect product $G_{n, m}=F_{N} \rtimes\left\langle\Phi^{n}, \Psi^{m}\right\rangle$ is word-hyperbolic. In this case the subgroups $\left\langle\varphi^{n}, \psi^{m}\right\rangle \subseteq \operatorname{Out}\left(F_{N}\right)$ and $\left\langle\Phi^{n}, \Psi^{m}\right\rangle \subseteq \operatorname{Aut}\left(F_{N}\right)$ are free of rank two and, as noted in Remark 4.18 below, the hyperbolicity of $G_{n, m}$ already implies that every non-trivial element of $\left\langle\varphi^{n}, \psi^{m}\right\rangle$ is hyperbolic.

Establishing the "purely iwip" part of Theorem 1.1 requires a much more delicate analysis and new tools and ideas, in order to rule out the existence of non-trivial reducible elements in free subgroups of $\operatorname{Out}\left(F_{N}\right)$ generated by two large powers of hyperbolic iwips. In particular, we exploit the interplay between the right ping-pong action of such subgroups on $\overline{\mathrm{cv}}_{N}$ and their simultaneous left ping-pong action on $\mathbb{P C u r r}\left(F_{N}\right)$. Thus ping-pong arguments play a key role in the proof Theorem 1.1. Note that ping-pong type arguments, in different settings, are also important in the proof of the Tits Alternative for $\operatorname{Out}\left(F_{N}\right)$ by Bestvina, Feighn and Handel [5, 6, 7]. Also, ping-pong arguments for iwips and the existence of Schottky-type free subgroups in $\operatorname{Out}\left(F_{N}\right)$ yielded by such arguments, are a key tool in the proof by Bridson and de la Harpe [11] that $\operatorname{Out}\left(F_{N}\right)$ is $C^{*}$-simple for $N \geq 3$. Both [5] and [11] use the ping-pong arguments (and their consequences) for the action of $\operatorname{Out}\left(F_{N}\right)$ on the set of the "legal" or "stable" laminations associated to all iwip elements of $\operatorname{Out}\left(F_{N}\right)$ and exploit the fact that this set admits an $\operatorname{Out}\left(F_{N}\right)$-equivariant embedding in $\overline{\mathrm{CV}}_{N}$.

A careful analysis of the proof of Theorem 1.1 shows that its conclusion holds for an arbitrary finite number of hyperbolic iwips:

Corollary 1.3. Let $\varphi_{1}, \ldots, \varphi_{k} \in \operatorname{Out}\left(F_{N}\right)$ be hyperbolic iwips such that for every $1 \leq i<j \leq k$ the subgroup $\left\langle\varphi_{i}, \varphi_{j}\right\rangle$ is not virtually cyclic. Then there exist $n_{1}, \ldots, n_{k} \geq 1$ such that the subgroup $G=\left\langle\varphi_{1}^{n_{1}}, \ldots, \varphi_{k}^{n_{k}}\right\rangle \subseteq \operatorname{Out}\left(F_{N}\right)$ is free of rank $k$, and such that every nontrivial element of $G$ is again a hyperbolic iwip.

An interesting goal for future work would be to develop a theory of "convex-cocompact" subgroups of $\operatorname{Out}\left(F_{N}\right)$ that resembles the theory of convex-cocompact subgroups of mapping class groups. A first step for such a theory is given in [36. We informally call the free subgroups of $\operatorname{Out}\left(F_{N}\right)$ generated by two large powers of hyperbolic iwips, that appear in the conclusion of Theorem 1.1, Shottky-type subgroups of $\operatorname{Out}\left(F_{N}\right)$. We believe 
that Schottky-type subgroups should provide basic examples of convexcocompact subgroups of of $\operatorname{Out}\left(F_{N}\right)$ and we hope that analyzing their properties will lead to a successful formulation of the convex-cocompactness theory in the $\operatorname{Out}\left(F_{N}\right)$ context.

Acknowledgement:

The authors are grateful to Chris Leininger for useful conversations. The authors also thank the referee for a careful reading of the paper and for many helpful suggestions.

\section{OUter SPACE AND THE SPACE OF GEODESIC CURRENTS}

We give here only a brief overview of basic facts related to Outer space and the space of geodesic currents. We refer the reader to [18, 31] for more detailed background information.

2.1. Outer space. Let $N \geq 2$. The unprojectivized Outer space $\mathrm{cv}_{N}$ consists of all minimal free and discrete isometric actions on $F_{N}$ on $\mathbb{R}$-trees (where two such actions are considered equal if there exists an $F_{N}$-equivariant isometry between the corresponding trees). There are several different topologies on $\mathrm{cv}_{N}$ that are known to coincide, in particular the equivariant GromovHausdorff convergence topology and the so-called axis or length function topology. Every $T \in \mathrm{cv}_{N}$ is uniquely determined by its translation length function $\|.\|_{T}: F_{N} \rightarrow \mathbb{R}$, where $\|g\|_{T}$ is the translation length of $g$ on $T$. Two trees $T_{1}, T_{2} \in \mathrm{cv}_{N}$ are close if the functions $\|\cdot\|_{T_{1}}$ and $\|\cdot\|_{T_{1}}$ are close pointwise on a large ball in $F_{N}$. The closure $\overline{\mathrm{cv}}_{N}$ of $\mathrm{cv}_{N}$ in either of these two topologies is well-understood and known to consists precisely of all the so-called very small minimal isometric actions of $F_{N}$ on $\mathbb{R}$-trees, see [4] and [14. The outer automorphism group $\operatorname{Out}\left(F_{N}\right)$ has a natural continuous right action on $\overline{\mathrm{cv}}_{N}$ (that leaves $\mathrm{cv}_{N}$ invariant) given at the level of length functions as follows: for $T \in \overline{\mathrm{cv}}_{N}$ and $\varphi \in \operatorname{Out}\left(F_{N}\right)$ we have $\|g\|_{T \varphi}=\|\Phi(g)\|_{T}$, with $g \in F_{N}$ and $\Phi \in \operatorname{Aut}\left(F_{N}\right)$ representing $\varphi \in \operatorname{Out}\left(F_{n}\right)$. In terms of tree actions, $T \varphi$ is equal to $T$ as a metric space, but the action of $F_{N}$ is modified to give $g_{T \varphi} x=\Phi(g)_{T} x$, where $x \in T, g \in F_{N}$ are arbitrary and where $\Phi \in \operatorname{Aut}\left(F_{N}\right)$ represents as before of the outer automorphism $\varphi$. The projectivized Outer space $\mathrm{CV}_{N}=\mathbb{P c v}_{N}$ is defined as the quotient $\mathrm{cv}_{N} / \sim$ where for $T_{1} \sim T_{2}$ whenever $T_{2}=c T_{1}$ for some $c>0$. One similarly defines the projectivization $\overline{\mathrm{CV}}_{N}=\mathbb{P} \overline{\mathrm{cV}}_{N}$ of $\overline{\mathrm{cv}}_{N}$ as $\overline{\mathrm{cV}}_{N} / \sim$ where $\sim$ is the same as above. The space $\overline{\mathrm{CV}}_{N}$ is compact and contains $\mathrm{CV}_{N}$ as a dense $\operatorname{Out}\left(F_{N}\right)$-invariant subset. The compactification $\overline{\mathrm{CV}}_{N}$ of $\mathrm{CV}_{N}$ is a free group analogue of the Thurston compactification of the Teichmüller space. For $T \in \overline{\mathrm{cv}}_{N}$ its $\sim$-equivalence class is denoted by $[T]$, so that $[T]$ is the image of $T$ in $\overline{\mathrm{CV}}_{N}$. The unprojectivized Outer space $\mathrm{cv}_{N}$ contains an $\operatorname{Out}\left(F_{N}\right)$-invariant closed subspace $c v_{N}^{1}$ which is $\operatorname{Out}\left(F_{N}\right)$-equivariantly homeomorphic to $\mathrm{CV}_{N}$. Namely, $c v_{N}^{1}$ consists of all trees $T \in \mathrm{cv}_{N}$ such that the quotient metric graph $T / F_{N}$ has volume one (that is, the sum of the 
lengths of its edges is equal to one). Many sources identify $\mathrm{CV}_{N}$ and $c v_{N}^{1}$ but we will distinguish these objects in the present paper.

2.2. Geodesic currents. Let $\partial^{2} F_{N}:=\left\{(x, y) \mid x, y \in \partial F_{N}, x \neq y\right\}$. The action of $F_{N}$ by translations on its hyperbolic boundary $\partial F_{N}$ defines a natural diagonal action of $F_{N}$ on $\partial^{2} F_{N}$. A geodesic current on $F_{N}$ is a positive Radon measure on $\partial^{2} F_{N}$ that is $F_{N}$-invariant and is also invariant under the "flip" map $\partial^{2} F_{N} \rightarrow \partial^{2} F_{N},(x, y) \mapsto(y, x)$. The space $\operatorname{Curr}\left(F_{N}\right)$ of all geodesic currents on $F_{N}$ has a natural $\mathbb{R}_{\geq 0}$-linear structure and is equipped with the weak ${ }^{*}$-topology of pointwise convergence on continuous functions. Every point $T \in \mathrm{cv}_{N}$ defines a simplicial chart on $\operatorname{Curr}\left(F_{N}\right)$ which allows one to think about geodesic currents as systems of nonnegative weights satisfying certain Kirchhoff-type equations; see [31 for details. We briefly recall the simplicial chart construction for the case where $T_{A} \in \mathrm{cv}_{N}$ is the Cayley tree corresponding to a free basis $A$ of $F_{N}$. For a nondegenerate geodesic segment $\gamma=[p, q]$ in $T_{A}$ the two-sided cylinder $C y l_{A}(\gamma) \subseteq \partial^{2} F_{N}$ consists of all $(x, y) \in \partial^{2} F_{N}$ such that the geodesic from $x$ to $y$ in $T_{A}$ passes through $\gamma=[p, q]$. Given a nontrivial freely reduced word $v \in F(A)=F_{N}$ and a current $\mu \in \operatorname{Curr}\left(F_{N}\right)$, the "weight" $\langle v, \mu\rangle_{A}$ is defined as $\mu\left(C y l_{A}(\gamma)\right)$ where $\gamma$ is any segment in the Cayley graph $T_{A}$ labelled by $v$ (the fact that the measure $\mu$ is $F_{N}$-invariant implies that a particular choice of $\gamma$ does not matter). A current $\mu$ is uniquely determined by a family of weights $\left(\langle v, \mu\rangle_{A}\right)_{v \in F_{N}-\{1\}}$. The weak*-topology on $\operatorname{Curr}\left(F_{N}\right)$ corresponds to pointwise convergence of the weights for every $v \in F_{N}, v \neq 1$.

There is a natural left action of $\operatorname{Out}\left(F_{N}\right)$ on $\operatorname{Curr}\left(F_{N}\right)$ by continuous linear transformations. Specifically, let $\mu \in \operatorname{Curr}\left(F_{N}\right), \varphi \in \operatorname{Out}\left(F_{N}\right)$ and let $\Phi \in \operatorname{Aut}\left(F_{N}\right)$ be a representative of $\varphi$ in $\operatorname{Aut}\left(F_{N}\right)$. Since $\Phi$ is a quasiisometry of $F_{N}$, it extends to a homeomorphism of $\partial F_{N}$ and, diagonally, defines a homeomorphism of $\partial^{2} F_{N}$. The measure $\varphi \mu$ on $\partial^{2} F_{N}$ is defined as follows. For a Borel subset $S \subseteq \partial^{2} F_{N}$ we have $(\varphi \mu)(S):=\mu\left(\Phi^{-1}(S)\right)$. One then checks that $\varphi \mu$ is a current and that it does not depend on the choice of a representative $\Phi$ of $\varphi$.

For every $g \in F_{N}, g \neq 1$ there is an associated counting current $\eta_{g} \in$ $\operatorname{Curr}\left(F_{N}\right)$. If $A$ is a free basis of $F_{N}$ and the conjugacy class $[g]$ of $g$ is realized by a "cyclic word" $W$ (that is a cyclically reduced word in $F(A)$ written on a circle with no specified base-vertex), then for every nontrivial freely reduced word $v \in F(A)=F_{N}$ the weight $\left\langle v, \eta_{g}\right\rangle_{A}$ is equal to the total number of occurrences of $v^{ \pm 1}$ in $W$ (where an occurrence of $v$ in $W$ is a vertex on $W$ such that we can read $v$ in $W$ clockwise without going off the circle). We refer the reader to 31 for a detailed exposition on the topic. By construction the counting current $\eta_{g}$ depends only on the conjugacy class $[g]$ of $[g]$ and it also satisfies $\eta_{g}=\eta_{g^{-1}}$. One can check [31] that for $\varphi \in \operatorname{Out}\left(F_{N}\right)$ and $g \in F_{N}, g \neq 1$ we have $\varphi \eta_{g}=\eta_{\varphi(g)}$. Scalar multiples $c \eta_{g} \in \operatorname{Curr}\left(F_{N}\right)$, where $c \geq 0, g \in F_{N}, g \neq 1$ are called rational currents. A key fact about $\operatorname{Curr}\left(F_{N}\right)$ states that the set of all rational currents is dense in $\operatorname{Curr}\left(F_{N}\right)$. 
The space of projectivized geodesic currents is defined as $\mathbb{P C u r r}\left(F_{N}\right)=$ $\operatorname{Curr}\left(F_{N}\right)-\{0\} / \sim$ where $\mu_{1} \sim \mu_{2}$ whenever there exists $c>0$ such that $\mu_{2}=c \mu_{1}$. The $\sim$-equivalence class of $\mu \in \operatorname{Curr}\left(F_{N}\right)-\{0\}$ is denoted by $[\mu]$. The action of $\operatorname{Out}\left(F_{N}\right)$ on $\operatorname{Curr}\left(F_{N}\right)$ descends to a continuous action of $\operatorname{Out}\left(F_{N}\right)$ on $\mathbb{P C u r r}\left(F_{N}\right)$. The space $\mathbb{P C u r r}\left(F_{N}\right)$ is compact and the set $\left\{\left[\eta_{g}\right] g \in F_{N}, g \neq 1\right\}$ is a dense subset of it.

2.3. Intersection form. In [34 we constructed a natural geometric intersection form which pairs trees and currents:

Proposition 2.1. 34] Let $N \geq 2$. There exists a unique continuous map $\langle\rangle:, \overline{c v}_{N} \times \operatorname{Curr}\left(F_{N}\right) \rightarrow \mathbb{R}_{\geq 0}$ with the following properties:

(1) We have $\left\langle T, c_{1} \mu_{1}+c_{2} \mu_{2}\right\rangle=c_{1}\left\langle T, \mu_{1}\right\rangle+c_{2}\left\langle T, \mu_{2}\right\rangle$ for any $T \in \overline{c v}_{N}$, $\mu_{1}, \mu_{2} \in \operatorname{Curr}\left(F_{N}\right), c_{1}, c_{2} \geq 0$.

(2) We have $\langle c T, \mu\rangle=c\langle T, \mu\rangle$ for any $T \in \overline{c v}_{N}, \mu \in \operatorname{Curr}\left(F_{N}\right)$ and $c \geq 0$.

(3) We have $\langle T \varphi, \mu\rangle=\langle T, \varphi \mu\rangle$ for any $T \in \overline{c v}_{N}, \mu \in \operatorname{Curr}\left(F_{N}\right)$ and $\varphi \in \operatorname{Out}\left(F_{N}\right)$.

(4) We have $\left\langle T, \eta_{g}\right\rangle=\|g\|_{T}$ for any $T \in \overline{c v}_{N}$ and $g \in F_{N}, g \neq 1$.

Note that here we work with the right action of $\operatorname{Out}\left(F_{N}\right)$ on $\overline{\mathrm{cv}}_{N}$, which is related to the left action of $\operatorname{Out}\left(F_{N}\right)$ on $\overline{\mathrm{cv}}_{N}$ considered in 34] via $T \varphi=$ $\varphi^{-1} T$, where $T \in \overline{\mathrm{cv}}_{N}, \varphi \in \operatorname{Out}\left(F_{N}\right)$. This accounts for the difference in how part (3) of Proposition 2.1 is stated above compared with the formulation of the main result in 34 .

\section{Stabilizers of Eigentrees AND EIGENCURRENTS}

Definition 3.1. An element $\varphi \in \operatorname{Out}\left(F_{N}\right)$ is reducible if there exists a free product decomposition $F_{N}=C_{1} * \ldots C_{k} * F^{\prime}$, where $k \geq 1$ and $C_{i} \neq\{1\}$, such that $\varphi$ permutes the conjugacy classes of subgroups $C_{1}, \ldots, C_{k}$ in $F_{N}$. An element $\varphi \in \operatorname{Out}\left(F_{N}\right)$ is called irreducible if it is not reducible. An element $\varphi \in \operatorname{Out}\left(F_{N}\right)$ is said to be irreducible with irreducible powers or an iwip for short, if for every $n \geq 1$ the power $\varphi^{n}$ is irreducible (sometimes such automorphisms are also called fully irreducible). This is equivalent to the property that no positive power of $\varphi$ fixes a conjugacy class of a proper free factor of $F_{N}$

An outer automorphism $\varphi \in \operatorname{Out}\left(F_{N}\right)$ is hyperbolic or atoroidal if no positive power of $\varphi$ fixes the conjugacy class of a nontrivial element of $F_{N}$.

An automorphism $\Phi \in \operatorname{Aut}\left(F_{N}\right)$, is called hyperbolic or atoroidal if the outer automorphism $\varphi \in \operatorname{Out}\left(F_{N}\right)$ defined by $\Phi$ is atoroidal.

A result of Brinkmann [12, together with the Combination Theorem of Bestvina and Feighn [1], implies that $\Phi \in \operatorname{Aut}\left(F_{N}\right)$ is atoroidal if and only if the mapping torus group $F_{N} \rtimes_{\Phi} \mathbb{Z}$ is word-hyperbolic.

The following result is due to Reiner Martin [44]: 
Proposition 3.2. Let $\varphi \in \operatorname{Out}\left(F_{N}\right)$ be a hyperbolic iwip. Then there exist unique $\left[\mu_{+}\right],\left[\mu_{-}\right] \in \mathbb{P} C \operatorname{curr}\left(F_{N}\right)$ with the following properties:

(1) The elements $\left[\mu_{+}\right],\left[\mu_{-}\right] \in \mathbb{P} C \operatorname{Curr}\left(F_{N}\right)$ are the only fixed points of $\varphi$ in $\mathbb{P} C u r r\left(F_{N}\right)$.

(2) For any $[\mu] \neq\left[\mu_{-}\right]$we have $\lim _{n \rightarrow \infty} \varphi^{n}[\mu]=\left[\mu_{+}\right]$and for any $[\mu] \neq$ $\left[\mu_{+}\right]$we have $\lim _{n \rightarrow \infty} \varphi^{-n}[\mu]=\left[\mu_{-}\right]$.

(3) We have $\varphi \mu_{+}=\lambda_{+} \mu_{+}$and $\varphi^{-1} \mu_{-}=\lambda_{-} \mu_{-}$where $\lambda_{+}>1$ and $\lambda_{-}>$ 1. Moreover $\lambda_{+}$is the Perron-Frobenius eigenvalue of any traintrack representative of $\varphi$ and $\lambda_{-}$is the Perron-Frobenius eigenvalue of any train-track representative of $\varphi^{-1}$.

Note that if $\varphi \in \operatorname{Out}\left(F_{N}\right)$ is a non-hyperbolic iwip, the conclusion of Proposition 3.2 still holds if $\operatorname{PCurr}\left(F_{N}\right)$ is replaced by the minimal set $\mathcal{M} \subseteq$ $\mathbb{P} \operatorname{Curr}\left(F_{N}\right)$, where $\mathcal{M}$ is the closure in $\operatorname{PCurr}\left(F_{N}\right)$ of the set of all $\left[\eta_{a}\right]$, where $a \in F_{N}$ is a primitive element (see [4]).

A similar statement is known for $\overline{\mathrm{CV}}_{N}$ by a result of Levitt and Lustig [41]:

Proposition 3.3. Let $\varphi \in \operatorname{Out}\left(F_{N}\right)$ be an iwip. Then there exist unique $\left[T_{+}\right],\left[T_{-}\right] \in \overline{C V}_{N}$ with the following properties:

(1) The elements $\left[T_{+}\right],\left[T_{-}\right] \in \overline{C V}_{N}$ are the only fixed points of $\varphi$ in $\overline{C V}_{N}$.

(2) For any $[T] \in \overline{C V}_{N},[T] \neq\left[T_{-}\right]$we have $\lim _{n \rightarrow \infty}\left[T \varphi^{n}\right]=\left[T_{+}\right]$and for any $[T] \in \overline{C V}_{N},[T] \neq\left[T_{+}\right]$we have $\lim _{n \rightarrow \infty}\left[T \varphi^{-n}\right]=\left[T_{-}\right]$.

(3) We have $T_{+} \varphi=\lambda_{+} T$ and $T_{-} \varphi^{-1}=\lambda_{-} T_{-}$where $\lambda_{+}>1$ and $\lambda_{-}>1$. Moreover $\lambda_{+}$is the Perron-Frobenius eigenvalue of any train-track representative of $\varphi$ and $\lambda_{-}$is the Perron-Frobenius eigenvalue of any train-track representative of $\varphi^{-1}$.

Moreover, in both [41] and [44] it is proved that the convergence to $\left[T_{+}\right]$ and $\left[\mu_{+}\right]$in the above statements is uniform on compact subsets. More precisely:

Proposition 3.4. Let $\varphi \in \operatorname{Out}\left(F_{N}\right)$ be a hyperbolic iwip. Let $\left[T_{+}\right],\left[T_{-}\right] \in$ $\overline{C V}_{N}$ and $\left[\mu_{+}\right],\left[\mu_{-}\right] \in \mathbb{P} C u r r\left(F_{N}\right)$ be as in Proposition 3.3 and Proposition 3.2 above.

Let $U, U^{\prime}$ be open neighborhoods in $\overline{C V}_{N}$ of $\left[T_{+}\right]$and $\left[T_{-}\right]$respectively and let $V, V^{\prime}$ be open neighborhoods in $\mathbb{P} C u r r\left(F_{N}\right)$ of $\left[\mu_{+}\right]$and $\left[\mu_{-}\right]$respectively. Then there exists a constant $M \geq 1$ such that for every $n \geq M$ we have $\left(\overline{C V}_{N}-U^{\prime}\right) \varphi^{n} \subseteq U$ and $\varphi^{n}\left(\mathbb{P} C u r r\left(F_{N}\right)-V^{\prime}\right) \subseteq V$.

Proposition 3.4 immediately implies, via the standard ping-pong argument, the following:

Corollary 3.5. Let $\varphi, \psi \in \operatorname{Out}\left(F_{N}\right)$ be hyperbolic iwips such that $\left[T_{ \pm}(\varphi)\right]$, $\left[T_{ \pm}(\psi)\right] \in \overline{C V}_{N}$ are four distinct points or that $\left[\mu_{ \pm}(\varphi)\right],\left[\mu_{ \pm}(\psi)\right] \in \mathbb{P} \operatorname{Curr}\left(F_{N}\right)$ are four distinct points. Then there exists $M \geq 1$ such that for every $m, n \geq M$ the subgroup $\left\langle\varphi^{m}, \psi^{n}\right\rangle \subseteq \operatorname{Out}\left(F_{N}\right)$ is free of rank two with free basis $\varphi^{m}, \psi^{n}$. 
We show in Section 4 below (specifically, see Proposition 4.10) that Proposition 3.4 can actually be formally derived from pointwise convergence to $\left[T_{+}\right]$and $\left[\mu_{+}\right]$in Proposition 3.3 and Proposition 3.2 . This will give an alternative proof of Proposition 3.4.

In 35. we gave a characterization of the situation where $\langle T, \mu\rangle=0$, in terms of the dual algebraic lamination $L^{2}(T)$ of the $\mathbb{R}$-tree $T$ and the support $\operatorname{Supp}(\mu)$ of the current $\mu$ (see [35] for a precise definition of these terms):

Theorem 3.6. 35] Let $T \in \overline{c v}_{N}$ and $\mu \in \operatorname{Curr}\left(F_{N}\right)$. Then $\langle T, \mu\rangle=0$ if and only if $\operatorname{Supp}(\mu) \subseteq L^{2}(T)$.

As a consequence, we proved [35]:

Proposition 3.7. 35] Let $\varphi \in \operatorname{Out}\left(F_{N}\right)$ be a hyperbolic iwip, and let $\left[T_{+}\right],\left[T_{-}\right] \in \overline{C V}_{N}$ and $\left[\mu_{+}\right],\left[\mu_{-}\right] \in \mathbb{P} \operatorname{Curr}\left(F_{N}\right)$ be as in Proposition 3.3 and Proposition 3.2 above. Then the following hold:

(1) For $T \in \overline{c v}\left(F_{N}\right)$ we have $\left\langle T, \mu_{+}\right\rangle=0$ if and only if $[T]=\left[T_{-}\right]$and we have $\left\langle T, \mu_{-}\right\rangle=0$ if and only if $[T]=\left[T_{+}\right]$.

(2) For $\mu \in \operatorname{Curr}\left(F_{N}\right), \mu \neq 0$ we have $\left\langle T_{+}, \mu\right\rangle=0$ if and only if $[\mu]=$ $\left[\mu_{-}\right]$and we have $\left\langle T_{-}, \mu\right\rangle=0$ if and only if $[\mu]=\left[\mu_{+}\right]$.

(3) In particular, we have $\left\langle T_{+}, \mu_{+}\right\rangle>0$ and $\left\langle T_{-}, \mu_{-}\right\rangle>0$.

Note also that, as a direct comparison of the definitions shows, if $\varphi \in$ $\operatorname{Out}\left(F_{N}\right)$ is a hyperbolic iwip, then $\operatorname{Supp}\left(\mu_{+}(\varphi)\right)$ is exactly what was termed the "stable lamination" $\Lambda_{\varphi}^{+}$of $\varphi$ in [5].

A result of [5], which is reproved in [37] via different methods, states:

Proposition 3.8. [5, 37] Let $N \geq 3$ and let $\varphi \in \operatorname{Out}\left(F_{N}\right)$ be an iwip. Then $\operatorname{Stab}_{\text {Out }\left(F_{N}\right)}\left(\left[T_{+}(\varphi)\right]\right)$ is virtually cyclic and contains $\langle\varphi\rangle$ as a subgroup of finite index.

As a consequence, we derive:

Proposition 3.9. Let $\varphi \in \operatorname{Out}\left(F_{N}\right)$ be a hyperbolic iwip. Then

$\operatorname{Stab}_{\text {Out }\left(F_{N}\right)}\left(\left[T_{+}(\varphi)\right]\right)=\operatorname{Stab}_{\text {Out }\left(F_{N}\right)}\left(\operatorname{Supp}\left(\mu_{-}(\varphi)\right)\right)=\operatorname{Stab}_{\text {Out }\left(F_{N}\right)}\left(\left[\mu_{-}(\varphi)\right]\right)$, and this stabilizer is virtually cyclic.

Proof. Proposition 3.7 implies that $\operatorname{Stab}_{O u t\left(F_{N}\right)}\left(\left[T_{+}\right]\right)=\operatorname{Stab}_{O u t}\left(F_{N}\right)\left(\left[\mu_{-}\right]\right)$. Indeed, suppose $\varphi \in \operatorname{Stab}_{O u t\left(F_{N}\right)}\left(\left[T_{+}\right]\right)$, so that $T_{+} \varphi=c T_{+}$for some $c>0$. Then

$$
\left\langle T_{+}, \varphi \mu_{-}\right\rangle=\left\langle T_{+} \varphi, \mu_{-}\right\rangle=\left\langle c T_{+}, \mu_{-}\right\rangle=c\left\langle T_{+}, \mu_{-}\right\rangle=0
$$

Therefore $\varphi\left[\mu_{-}\right]=\left[\mu_{-}\right]$by part 2 of Proposition 3.7 and hence $\operatorname{Stab}_{\text {Out }\left(F_{N}\right)}\left(\left[T_{+}\right]\right) \subseteq$ $\operatorname{Stab}_{O u t\left(F_{N}\right)}\left(\left[\mu_{-}\right]\right)$. A similar argument using part (1) of Proposition 3.7 shows that $\operatorname{Stab}_{O u t\left(F_{N}\right)}\left(\left[\mu_{-}\right]\right) \subseteq \operatorname{Stab}_{O u t\left(F_{N}\right)}\left(\left[T_{+}\right]\right)$, and hence $\operatorname{Stab}_{O u t\left(F_{N}\right)}\left(\left[T_{+}\right]\right)=$ $\operatorname{Stab}_{\text {Out }\left(F_{N}\right)}\left(\left[\mu_{-}\right]\right)$.

We claim that $\operatorname{Stab}_{O u t\left(F_{N}\right)}\left(\operatorname{Supp}\left(\mu_{-}\right)\right)=\operatorname{Stab}_{O u t\left(F_{N}\right)}\left(\left[\mu_{-}\right]\right)$. Indeed, the inclusion $\operatorname{Stab}_{\text {Out }\left(F_{N}\right)}\left(\left[\mu_{-}\right]\right) \subseteq \operatorname{Stab}_{\text {Out }\left(F_{N}\right)}\left(\operatorname{Supp}\left(\mu_{-}\right)\right)$is obvious. 
Suppose now that $\varphi \in \operatorname{Stab}_{O u t\left(F_{N}\right)}\left(\operatorname{Supp}\left(\mu_{-}\right)\right)$. Then $\operatorname{Supp}\left(\varphi \mu_{-}\right)=$ $\operatorname{Supp}\left(\mu_{-}\right)$.

From Proposition 3.7 we know that $\left\langle T_{+}, \mu_{-}\right\rangle=0$. Thus we derive from Theorem 3.6 that $\operatorname{Supp}\left(\varphi \mu_{-}\right)=\operatorname{Supp}\left(\mu_{-}\right)$is contained in the dual lamination $L^{2}\left(T_{+}\right)$of $T_{+}$, so that the converse implication of Theorem 3.6 implies that $\left\langle T_{+}, \varphi \mu_{-}\right\rangle=0$. Therefore, by part (2) of Proposition 3.7, we have $\varphi\left[\mu_{-}\right]=\left[\mu_{-}\right]$. Thus $\operatorname{Stab}_{O u t\left(F_{N}\right)}\left(\operatorname{Supp}\left(\mu_{-}\right)\right) \subseteq \operatorname{Stab}_{O u t\left(F_{N}\right)}\left(\left[\mu_{-}\right]\right)$and hence $\operatorname{Stab}_{\text {Out }\left(F_{N}\right)}\left(\operatorname{Supp}\left(\mu_{-}\right)\right)=\operatorname{Stab}_{O u t\left(F_{N}\right)}\left(\left[\mu_{-}\right]\right)$, as claimed.

In [5] it is first proved that for an iwip $\varphi$ the stabilizer $\operatorname{Stab}_{O u t\left(F_{N}\right)}\left(\Lambda_{\varphi}^{+}\right)$ is virtually cyclic and then that $\operatorname{Stab}_{\text {Out }\left(F_{N}\right)}\left(\Lambda_{\varphi}^{+}\right)=\operatorname{Stab}_{\text {Out }\left(F_{N}\right)}\left(\left[T_{+}(\varphi)\right]\right)$. Proposition 3.9 above recovers these results, for a hyperbolic iwip $\varphi$, as a consequence of Proposition 3.8 about virtual cyclicity of $\operatorname{Stab}_{O u t\left(F_{N}\right)}\left(\left[T_{+}(\varphi)\right]\right)$, for which [37] provided an alternative proof to the argument given in [5].

Proposition 3.10. Let $G \subseteq \operatorname{Out}\left(F_{N}\right)$ be a subgroup and such that there exists a hyperbolic iwip $\varphi \in G$. Let $\left[T_{+}(\varphi)\right],\left[T_{-}(\varphi)\right] \in \overline{C V}_{N},\left[\mu_{+}(\varphi)\right],\left[\mu_{-}(\varphi)\right] \in$ $\mathbb{P} C \operatorname{urr}\left(F_{N}\right)$ be the attracting and repelling fixed points of $g$ in $\overline{C V}_{N}$ and $\mathbb{P} C u r r\left(F_{N}\right)$ accordingly. Then exactly one of the following occurs:

(1) The group $G$ is virtually cyclic and preserves the sets $\left\{\left[T_{+}(\varphi)\right]\right.$, $\left.\left[T_{-}(\varphi)\right]\right\} \subseteq \overline{C V}_{N},\left\{\left[\mu_{+}(\varphi)\right],\left[\mu_{-}(\varphi)\right]\right\} \subseteq \mathbb{P} \operatorname{Curr}\left(F_{N}\right)$.

(2) The group $G$ contains a hyperbolic iwip $\psi=g \varphi g^{-1}$ for some $g \in G$ such that $\left\{\left[T_{+}(\varphi)\right],\left[T_{-}(\varphi)\right]\right\} \cap\left\{\left[T_{+}(\psi)\right],\left[T_{-}(\psi)\right]\right\}=\emptyset$ and $\left\{\left[\mu_{+}(\varphi)\right],\left[\mu_{-}(\varphi)\right]\right\} \cap$ $\left\{\left[\mu_{+}(\psi)\right],\left[\mu_{-}(\psi)\right]\right\}=\emptyset$. Moreover, in this case there exists $M \geq 1$ such that the subgroup $\left\langle\varphi^{M}, \psi^{M}\right\rangle \subseteq G$ is free of rank two.

Proof. Recall that by Proposition 3.9 we have $\operatorname{Stab}_{O u t\left(F_{N}\right)}\left[T_{+}(\varphi)\right]=$ $\operatorname{Stab}_{O u t\left(F_{N}\right)}\left[\mu_{-}(\varphi)\right]$ and $\operatorname{Stab}_{O u t\left(F_{N}\right)}\left[T_{-}(\varphi)\right]=\operatorname{Stab}_{O u t\left(F_{N}\right)}\left[\mu_{+}(\varphi)\right]$ and both of these are virtually cyclic and contain $\langle\varphi\rangle$ as a subgroup of finite index.

If $G$ preserves the set $\left\{\left[T_{+}(\varphi)\right],\left[T_{-}(\varphi)\right]\right\}$, then $G$ has a subgroup of index at most 2 that fixes each of $\left[T_{ \pm}(\varphi)\right]$ and hence $G$ is virtually cyclic. Thus we may assume that $G$ does not preserve $\left\{\left[T_{+}(\varphi)\right],\left[T_{-}(\varphi)\right]\right\}$. So there exists $g \in G$ such that $\left[T_{+}(\varphi)\right] g \notin\left\{\left[T_{+}(\varphi)\right],\left[T_{-}(\varphi)\right]\right\}$ or that $\left[T_{-}(\varphi)\right] g \notin$ $\left\{\left[T_{+}(\varphi)\right],\left[T_{-}(\varphi)\right]\right\}$. We assume the former as the other case is symmetric. Thus $\left[T_{+}(\varphi)\right] g \neq\left[T_{ \pm}(\varphi)\right]$. Note that $\psi=g^{-1} \varphi g \in G$ is also an atoriodal iwip and that $\left[T_{+}(\psi)\right]=\left[T_{+}(\varphi)\right] g$. We claim that $\left[T_{-}(\varphi)\right] \neq\left[T_{ \pm}(\varphi)\right] g$. Indeed, otherwise we have $\left[T_{-}\left(g^{-1} \varphi g\right)\right]=\left[T_{ \pm}(\varphi)\right]$ and hence $g^{-1} \varphi g \in$ $\operatorname{Stab}_{O u t\left(F_{N}\right)}\left[T_{+}(\varphi)\right]$ or $g^{-1} \varphi g \in \operatorname{Stab}_{O u t\left(F_{N}\right)}\left[T_{-}(\varphi)\right]$. In either case (since both stabilizers contain $\langle\varphi\rangle$ as subgroup of finite index) $g^{-1} \varphi^{k} g=\varphi^{l}$ for some $k \neq 0, l \neq 0$ and therefore $g^{-1} \varphi^{k} g$ has the same fixed points in $\overline{\mathrm{CV}}_{N}$ as does $\varphi^{l}$, namely, $\left[T_{ \pm}(\varphi)\right]$. This contradicts the fact that $g^{-1} \varphi g$ fixes the point $\left[T_{+}(\varphi)\right] g \neq\left[T_{ \pm}(\varphi)\right]$. Thus $\left[T_{ \pm}(\varphi)\right],\left[T_{ \pm}(\psi)\right]$ are four distinct points. Therefore, by Corollary [3.5, sufficiently high powers $\varphi^{M}, \psi^{M}$ freely generate a free subgroup of rank two in $G$, as required. Note that in this case we also have that $\left[\mu_{ \pm}(\varphi)\right],\left[\mu_{ \pm}(\psi)\right]$ are four distinct points by Proposition 3.7 . 
Corollary 3.11. Let $\varphi, \psi \in \operatorname{Out}\left(F_{N}\right)$ be hyperbolic iwips. Then the following conditions are equivalent:

(1) The subgroup $\langle\varphi, \psi\rangle \subseteq \operatorname{Out}\left(F_{N}\right)$ is not virtually cyclic.

(2) There exist $m, n \geq 1$ such that $\left\langle\varphi^{m}, \psi^{n}\right\rangle$ is free of rank two.

(3) We have $\langle\varphi\rangle \cap\langle\psi\rangle=\{1\}$.

(4) The points $\left[T_{ \pm}(\varphi)\right],\left[T_{ \pm}(\psi)\right]$ are four distinct elements in $\overline{C V}_{N}$.

(5) The points $\left[\mu_{ \pm}(\varphi)\right],\left[\mu_{ \pm}(\psi)\right]$ are four distinct elements in $\mathbb{P} C u r r\left(F_{N}\right)$.

Proof. It is obvious that (2) implies (1) and that (2) implies (3).

Suppose that (3) holds. We claim that $\left[T_{ \pm}(\varphi)\right],\left[T_{ \pm}(\psi)\right]$ are four distinct points in $\overline{\mathrm{CV}}_{N}$. Indeed, suppose that one of $\left[T_{ \pm}(\varphi)\right]$ is equal to one of $\left[T_{ \pm}(\psi)\right]$. This means that both $\varphi$ and $\psi$ have a common fixed point in $\overline{\mathrm{CV}}_{N}$ which is a pole of a hyperbolic iwip. The $\operatorname{Out}\left(F_{N}\right)$-stabilizer of that point is virtually infinite cyclic by Proposition 3.9, which implies that $\varphi$ and $\psi$ have some equal nonzero powers, contradicting the assumption $\langle\varphi\rangle \cap\langle\psi\rangle=$ $\{1\}$. Thus $\left[T_{ \pm}(\varphi)\right],\left[T_{ \pm}(\psi)\right]$ are four distinct points in $\overline{\mathrm{CV}}_{N}$. Therefore by Corollary 3.5 below, sufficiently high powers $\varphi^{M}, \psi^{M}$ freely generate a free subgroup of rank two in $\operatorname{Out}\left(F_{N}\right)$, so that (2) holds. Thus (3) implies (2) and, therefore (2) is equivalent to (3).

Suppose now that (1) holds. Suppose that (3) fails and there exist nonzero $n, m$ such that $\varphi^{n}=\psi^{m}$. Since $\varphi$ has the same fixed points in $\overline{\mathrm{CV}}_{N}$ as $\varphi^{n}$ and since $\psi$ has the same fixed points in $\overline{\mathrm{CV}}_{N}$ as $\psi^{n}$, it follows that $\varphi$ and $\psi$ have a common fixed point in $\overline{\mathrm{CV}}_{N}$. Therefore $\langle\varphi, \psi\rangle$ is contained in the $\operatorname{Out}\left(F_{N}\right)$-stabilizer of that point, which, by Proposition 3.9, is virtually cyclic. This implies that $\langle\varphi, \psi\rangle$ is virtually cyclic, contrary to our assumption (1). Thus (1) implies (3), which shows that (1), (2) and (3) are equivalent.

The fact that (4) and (5) are equivalent follows from Proposition 3.7. The proof that (3) implies (2) above also shows that (3) implies (4). The fact that (4) implies (2) follows from Corollary 3.5. This completes the proof.

Corollary 3.12. Let $\varphi \in \operatorname{Out}\left(F_{N}\right)$ be a hyperbolic iwip and let $\left[T_{ \pm}\right]$be the attracting and repelling fixed points of $\varphi$ in $\overline{C V}_{N}$. Then $\operatorname{Stab}_{O u t\left(F_{N}\right)}\left(\left[T_{+}\right]\right)=$ $\operatorname{Stab}_{\text {Out }\left(F_{N}\right)}\left(\left[T_{-}\right]\right)$.

Proof. Suppose there exists $g \in \operatorname{Stab}_{\text {Out }\left(F_{N}\right)}\left(\left[T_{+}\right]\right)$such that $g \notin \operatorname{Stab}_{O u t\left(F_{N}\right)}\left(\left[T_{-}\right]\right)$. Thus $\left[T_{-}\right] g \neq\left[T_{-}\right]$. Since $g$ fixes $T_{+}$, and since $g$ is a homeomorphism of $\overline{\mathrm{CV}}_{N}$, it follows that $\left[T_{-}\right] g \neq\left[T_{+}\right]$and hence $\left\{\left[T_{-}\right],\left[T_{+}\right]\right\} g \neq\left\{\left[T_{-}\right],\left[T_{+}\right]\right\}$. Put $G=\langle g, \psi\rangle$. Then $G$ does not leave the set $\left\{\left[T_{-}\right],\left[T_{+}\right]\right\}$invariant and hence $G$ contains a free subgroup of rank two by Proposition 3.10 and by Corollary 4.14. On the other hand $G \subseteq \operatorname{Stab}_{\text {Out }\left(F_{N}\right)}\left(\left[T_{+}\right]\right)$and hence $G$ is virtually cyclic, yielding a contradiction.

Thus $\operatorname{Stab}_{O u t\left(F_{N}\right)}\left(\left[T_{+}\right]\right) \subseteq \operatorname{Stab}_{O u t\left(F_{N}\right)}\left(\left[T_{-}\right]\right)$and hence, by symmetry, we also have $\operatorname{Stab}_{\text {Out }\left(F_{N}\right)}\left(\left[T_{-}\right]\right) \subseteq \operatorname{Stab}_{O u t}\left(F_{N}\right)\left(\left[T_{+}\right]\right)$.

Therefore $\operatorname{Stab}_{O u t\left(F_{N}\right)}\left(\left[T_{+}\right]\right)=\operatorname{Stab}_{O u t\left(F_{N}\right)}\left(\left[T_{-}\right]\right)$, as required. 
Note that, in Corollary 3.12 , if $\left[\mu_{ \pm}\right]$are the fixed points of $\varphi$ in $\mathbb{P C u r r}\left(F_{N}\right)$, then Corollary 3.12 and Proposition 3.9 imply that we in fact have

$\operatorname{Stab}_{\text {Out }\left(F_{N}\right)}\left(\left[T_{+}\right]\right)=\operatorname{Stab}_{O u t\left(F_{N}\right)}\left(\left[\mu_{-}\right]\right)=\operatorname{Stab}_{O u t\left(F_{N}\right)}\left(\left[T_{-}\right]\right)=\operatorname{Stab}_{O u t\left(F_{N}\right)}\left(\left[\mu_{+}\right]\right)$.

4. North-South Dynamics, Standard neighborhoods And height FUNCTIONS

Convention 4.1. For the remainder of this section, unless specified otherwise, let $\varphi \in \operatorname{Out}\left(F_{N}\right)$, where $N \geq 3$, be a hyperbolic iwip and let $\left[\mu_{+}\right],\left[\mu_{-}\right] \in \mathbb{P C u r r}\left(F_{N}\right),\left[T_{+}\right],\left[T_{-}\right] \in \overline{\mathrm{CV}}_{N}$ and $\lambda_{+}>1, \lambda_{-}>1$ be as in Proposition 3.2 and Proposition 3.3 .

Throughout this section we fix the (arbitrarily chosen) non-projectivized representatives $T_{+}$of $\left[T_{+}\right], T_{-}$of $\left[T_{-}\right], \mu_{+}$of $\left[\mu_{+}\right]$and $\mu_{-}$of $\left[\mu_{-}\right]$.

Definition 4.2 (Standard Neighborhoods). Let

$$
U_{+}=\left\{[\mu] \in \mathbb{P} \operatorname{Curr}\left(F_{N}\right):\left\langle T_{-}, \mu\right\rangle<\left\langle T_{+}, \mu\right\rangle\right\}
$$

and

$$
U_{-}=\left\{[\mu] \in \mathbb{P} \operatorname{Curr}\left(F_{N}\right):\left\langle T_{-}, \mu\right\rangle>\left\langle T_{+}, \mu\right\rangle\right\} .
$$

Note that by Proposition 3.7 for any $[\mu] \neq\left[\mu_{ \pm}\right]$we have $\left\langle T_{+}, \mu\right\rangle>0$ and $\left\langle T_{-}, \mu\right\rangle>0$. Therefore the following function is well-defined:

Definition 4.3 (Height function). Define

$$
f: \mathbb{P C u r r}\left(F_{N}\right)-\left\{\left[\mu_{+}\right],\left[\mu_{-}\right]\right\} \rightarrow \mathbb{R}
$$

as

$$
f([\mu]):=\log \frac{\left\langle T_{+}, \mu\right\rangle}{\left\langle T_{-}, \mu\right\rangle} .
$$

It is clear that $f: \mathbb{P} \operatorname{Curr}\left(F_{N}\right)-\left\{\left[\mu_{+}\right],\left[\mu_{-}\right]\right\} \rightarrow \mathbb{R}$ is continuous and, moreover, if we put $f\left(\left[\mu_{+}\right]\right)=\infty$ and $f\left(\left[\mu_{-}\right]\right)=-\infty$, this gives a continuous extension of $f$ to $\bar{f}: \mathbb{P} \operatorname{Curr}\left(F_{N}\right) \rightarrow \mathbb{R} \cup\{ \pm \infty\}$. We call $\bar{f}$ the extended height function. Note that $U_{+}=(\bar{f})^{-1}((0, \infty])$ and $U_{-}=(\bar{f})^{-1}([-\infty, 0))$.

Lemma 4.4. For any $[\mu] \neq\left[\mu_{ \pm}\right]$we have

$$
f(\varphi[\mu])=f([\mu])+\log \left(\lambda_{+} \lambda_{-}\right) .
$$

Proof. Let $[\mu] \neq\left[\mu_{ \pm}\right]$. Then

$$
\begin{gathered}
f(\varphi[\mu])=\log \frac{\left\langle T_{+}, \varphi \mu\right\rangle}{\left\langle T_{-}, \varphi \mu\right\rangle}=\log \frac{\left\langle T_{+} \varphi, \mu\right\rangle}{\left\langle T_{-} \varphi, \mu\right\rangle}= \\
=\log \frac{\left\langle\lambda_{+} T_{+}, \mu\right\rangle}{\left\langle\lambda_{-}^{-1} T_{-}, \mu\right\rangle}=\log \left(\lambda_{+} \lambda_{-} \frac{\left\langle T_{+}, \mu\right\rangle}{\left\langle T_{-}, \mu\right\rangle}\right)=f([\mu])+\log \left(\lambda_{+} \lambda_{-}\right) .
\end{gathered}
$$

The continuity of the intersection form and Proposition 3.7 imply that $U_{+}, U_{-}$are disjoint open subsets of $\mathbb{P C u r r}\left(F_{N}\right)$ and that $\left[\mu_{+}\right] \in U_{+}$and $\left[\mu_{-}\right] \in U_{-}$. 
Lemma 4.5. We have $\varphi\left(U_{+}\right) \subseteq U_{+}$and $\varphi^{-1}\left(U_{-}\right) \subseteq U_{-}$.

Proof. Let $[\mu] \in U_{+}$so that $\left\langle T_{-}, \mu\right\rangle\left\langle\left\langle T_{+}, \mu\right\rangle\right.$. We have $\left\langle T_{-}, \varphi \mu\right\rangle=$ $\left\langle T_{-} \varphi, \mu\right\rangle=\left\langle\frac{1}{\lambda_{-}} T_{-}, \mu\right\rangle=\frac{1}{\lambda_{-}}\left\langle T_{-}, \mu\right\rangle$. Similarly, $\left\langle T_{+}, \varphi \mu\right\rangle=\left\langle T_{+} \varphi, \mu\right\rangle=$ $\lambda_{+}\left\langle T_{+}, \mu\right\rangle$. Hence

$$
\left\langle T_{-}, \varphi \mu\right\rangle=\frac{1}{\lambda_{-}}\left\langle T_{-}, \mu\right\rangle \leq\left\langle T_{-}, \mu\right\rangle\left\langle\left\langle T_{+}, \mu\right\rangle \leq \lambda_{+}\left\langle T_{+}, \mu\right\rangle=\left\langle T_{+}, \varphi \mu\right\rangle\right.
$$

so that $[\varphi \mu] \in U_{+}$by definition of $U_{+}$. The proof that $\varphi^{-1}\left(U_{-}\right) \subseteq U_{-}$is symmetric.

Note that Lemma 4.5 implies that $\varphi\left(\bar{U}_{+}\right) \subseteq \bar{U}_{+}$and $\varphi^{-1}\left(\bar{U}_{-}\right) \subseteq \bar{U}_{-}$.

Lemma 4.6. We have:

(1) $\cap_{n=1}^{\infty} \varphi^{n}\left(\bar{U}_{+}\right)=\left\{\left[\mu_{+}\right]\right\}$and $\cap_{n=1}^{\infty} \varphi^{-n}\left(\bar{U}_{-}\right)=\left\{\left[\mu_{-}\right]\right\}$.

(2) For any neighborhood $V$ of $\left[\mu_{+}\right]$there exists $n \geq 1$ such that $\varphi^{n}\left(U_{+}\right) \subseteq$ $V$ and for any neighborhood $V^{\prime}$ of $\left[\mu_{-}\right]$there exists $n \geq 1$ such that $\varphi^{-n}\left(U_{-}\right) \subseteq V^{\prime}$.

Proof. (1) Since $\left[\mu_{+}\right]$is fixed by $\varphi$, it follows that $\left[\mu_{+}\right] \in \varphi^{n}\left(U_{+}\right)$for every $n \geq 1$ so that $\left[\mu_{+}\right] \in \cap_{n=1}^{\infty} \varphi^{n} U_{+}$. Suppose there exists $[\mu] \in \cap_{n=1}^{\infty} \varphi^{n} \bar{U}_{+}$ such that $[\mu] \neq\left[\mu_{+}\right]$. Let $\left[\mu_{n}\right]=\varphi^{-n}[\mu]$ for $n \geq 1$. Thus $\left[\mu_{n}\right] \in \bar{U}_{+}$for every $n \geq 1$. On the other hand $[\mu] \neq\left[\mu_{+}\right]$implies that $\lim _{n \rightarrow \infty} \varphi^{-n}[\mu]=$ $\left[\mu_{-}\right]$. Since $U_{-}$is open neighborhood of $\left[\mu_{-}\right]$, there exists $n_{0} \geq 1$ such that $\left[\mu_{n_{0}}\right]=\varphi^{-n_{0}}[\mu] \in U_{-}$. Thus $\mu_{n_{0}} \in \bar{U}_{+} \cap U_{-}$which contradicts the fact that $\bar{U}_{+} \subseteq\left\{[\nu] \in \mathbb{P} \operatorname{Curr}\left(F_{N}\right):\left\langle T_{-}, \nu\right\rangle \leq\left\langle T_{+}, \nu\right\rangle\right\}$ and $U_{-}$are disjoint. This establishes part (1) of the lemma.

(2) Let $V$ be an open neighborhood of $\left[\mu_{+}\right]$and suppose that there is no $n \geq 1$ such that $\varphi^{n}\left(U_{+}\right) \subseteq V$. Then there exists a sequence $\left[\mu_{n}\right] \in$ $\varphi^{n}\left(U_{+}\right)-V$. After passing to a subsequence, we have $\left[\mu_{n_{i}}\right] \rightarrow[\mu]$ as $i \rightarrow \infty$. Since $\operatorname{PCurr}\left(F_{N}\right)-V$ is closed, we have $[\mu] \notin V$. Since $\left[\mu_{n_{i}}\right] \in \varphi^{n_{i}}\left(\bar{U}_{+}\right)$and since the closed sets $\varphi^{n}\left(\bar{U}_{+}\right)$are nested, it follows that $[\mu] \in \cap_{n \geq 1} \varphi^{n}\left(\bar{U}_{+}\right)$. Therefore by part (1) we have $[\mu]=\left[\mu_{+}\right]$, which contradicts the fact that $[\mu] \notin V$.

Lemma 4.7. For any neighborhoods $U$ of $\left[\mu_{+}\right]$and $V$ of $\left[\mu_{-}\right]$there exists $M \geq 1$ such that for every $n \geq M$ we have $\varphi^{n}\left(\mathbb{P} C u r r\left(F_{N}\right)-V\right) \subseteq U$ and $\varphi^{-n}\left(\mathbb{P} C u r r\left(F_{N}\right)-U\right) \subseteq V$.

Proof. By applying Lemma 4.6 and making $U, V$ smaller if necessary, we may assume that $U$ and $V$ are disjoint open neighborhoods of $\left[\mu_{+}\right]$and $\left[\mu_{-}\right]$ accordingly such that $\varphi(U) \subseteq U$ and $\varphi^{-1}(V) \subseteq V$. Let $K=\mathbb{P} \operatorname{Curr}\left(F_{N}\right)-$ $(U \cup V)$. Thus $K$ is a compact subset of $\mathbb{P} \operatorname{Curr}\left(F_{N}\right)$. Since $\left[\mu_{-}\right] \notin K$, part (2) of Proposition 3.2 implies that

$$
K \subseteq \cup_{n=1}^{\infty} \varphi^{-n}(U) .
$$

Since the sets $\varphi^{-n}(U)$ are open and $K$ is compact, there exists $p \geq 1$ such that $K \subseteq \cup_{n=1}^{p} \varphi^{-n}\left(U_{+}\right)$. The assumption that $\varphi(U) \subseteq U$ implies that 
$\cup_{n=1}^{p} \varphi^{-n}(U)=\varphi^{-p}(U)$. Thus $K \subseteq \varphi^{-p}(U)$, so that $\varphi^{p}(K) \subseteq U_{+}$. Since $\varphi^{n}(U) \subseteq U$ for every $n \geq 1$, it follows that $\varphi^{n}(K) \subseteq U$ for every $n \geq p+1$ and, obviously $\varphi^{n}(U) \subseteq U$ for every $n \geq p+1$. Hence $\varphi^{n}\left(\mathbb{P C u r r}\left(F_{N}\right)-V\right) \subseteq$ $U$ for every $n \geq p+1$, as required. The argument for $\varphi^{-n}$ is symmetric.

Corollary 4.8. The group $\langle\varphi\rangle$ acts properly discontinuously and co-compactly on $\mathbb{P} C u r r\left(F_{N}\right)-\left\{\left[\mu_{+}\right],\left[\mu_{-}\right]\right\}$.

Proof. Let $K \subseteq \mathbb{P C u r r}\left(F_{N}\right)-\left\{\left[\mu_{+}\right],\left[\mu_{-}\right]\right\}$be a compact subset. Choose open neighborhoods $U_{+}$of $\left[\mu_{+}\right]$and $U_{-}$of $\left[\mu_{-}\right]$in $\operatorname{PCurr}\left(F_{N}\right)$ so that the sets $U_{+}, U_{-}, K$ are pairwise disjoint. Let $M \geq 1$ be as in Lemma 4.7 . Therefore for any $n \in \mathbb{Z}$ with $|n| \geq M$ we have $K \cap \varphi^{n} K=\emptyset$ since $\varphi^{n}(K) \subseteq$ $U_{+} \cup U_{-}$. This shows that $\langle\varphi\rangle$ acts properly discontinuously on $\mathbb{P} \operatorname{Curr}\left(F_{N}\right)-$ $\left\{\left[\mu_{+}\right],\left[\mu_{-}\right]\right\}$.

To see that the action of $\langle\varphi\rangle$ on $\mathbb{P C u r r}\left(F_{N}\right)-\left\{\left[\mu_{+}\right],\left[\mu_{-}\right]\right\}$is co-compact, put

$$
K:=\left\{[\mu] \in \mathbb{P} \operatorname{Curr}\left(F_{N}\right)-\left\{\left[\mu_{+}\right],\left[\mu_{-}\right]\right\} \mid 0 \leq f(\mu) \leq \log \left(\lambda_{+} \lambda_{-}\right)\right\}
$$

where $f$ is the height function as in Definition 4.3. The set $K$ is compact since the extended height function $\bar{f}: \mathbb{P C u r r}\left(F_{N}\right) \rightarrow \mathbb{R} \cup\{ \pm \infty\}$ is continuous and $K=(\bar{f})^{-1}\left(\left[0, \log \left(\lambda_{+} \lambda_{-}\right)\right]\right)$. Lemma 4.4 implies that $\langle\varphi\rangle K=\mathbb{P} \operatorname{Curr}\left(F_{N}\right)-\left\{\left[\mu_{+}\right],\left[\mu_{-}\right]\right\}$. Thus the action of $\langle\varphi\rangle$ on $\mathbb{P} \operatorname{Curr}\left(F_{N}\right)-$ $\left\{\left[\mu_{+}\right],\left[\mu_{-}\right]\right\}$is co-compact, as required.

We summarize the preceding results in the following:

Proposition 4.9. Let $\varphi, T_{+}, T_{-}, \mu_{+}, \mu_{-}$be as in Convention 4.1. Let $U_{+}, U_{-}$be as in Definition 4.2. Then the following hold:

(1) $U_{+}$is an open neighborhood of $\left[\mu_{+}\right]$and $U_{-}$is an open neighborhood of $\left[\mu_{-}\right]$in $\mathbb{P} C u r r\left(F_{N}\right)$ such that $\varphi\left(U_{+}\right) \subseteq U_{+}$and $\varphi^{-1}\left(U_{-}\right) \subseteq U_{-}$.

(2) $\cap_{n \geq 1} \varphi^{n}\left(\bar{U}_{+}\right)=\left\{\left[\mu_{+}\right]\right\}$and $\cap_{n \geq 1} \varphi^{-n}\left(\bar{V}_{-}\right)=\left\{\left[\mu_{-}\right]\right\}$.

(3) For any neighborhood $U$ of $\left[\mu_{+}\right]$there exists $n \geq 1$ such that $\varphi^{n}\left(U_{+}\right) \subseteq$ $U$ and for any neighborhood $U^{\prime}$ of $\left[\mu_{-}\right]$there is $n \geq 1$ such that $\varphi^{-n}\left(U_{-}\right) \subseteq U^{\prime}$

(4) For any neighborhoods $U$ of $\left[\mu_{+}\right]$and $U^{\prime}$ of $\left[\mu_{-}\right]$in $\mathbb{P} C u r r\left(F_{N}\right)$ there exists $M \geq 1$ such that for every $n \geq M$ we have $\varphi^{n}\left(\mathbb{P} C u r r\left(F_{N}\right)-\right.$ $\left.U^{\prime}\right) \subseteq U$ and $\varphi^{-n}\left(\mathbb{P} C u r r\left(F_{N}\right)-U\right) \subseteq U^{\prime}$.

(5) The action of $\langle\varphi\rangle$ on $\mathbb{P} C u r r\left(F_{N}\right)-\left\{\left[\mu_{+}\right],\left[\mu_{-}\right]\right\}$is properly discontinuous and cocompact.

A symmetric argument gives analogous statements for neighborhoods of $\left[T_{+}\right]$and $\left[T_{-}\right]$in $\overline{\mathrm{CV}}_{N}$. (The only difference is that in the proofs the result of Reiner Martin about the North-South dynamics for the action of $\varphi$ on $\mathbb{P} \operatorname{Curr}\left(F_{N}\right)$ has to be replaced by the corresponding result of Levitt-Lustig about the North-South dynamics for the action of $\varphi$ on $\left.\overline{\mathrm{CV}}_{N}\right)$.

We summarize them in the following: 
Proposition 4.10. Let $\varphi, T_{+}, T_{-}, \mu_{+}, \mu_{-}$be as in Convention 4.1. Define

$$
\begin{aligned}
& V_{+}=\left\{[T] \in \overline{C V}_{N}:\left\langle T, \mu_{-}\right\rangle<\left\langle T, \mu_{+}\right\rangle\right\}, \\
& V_{-}=\left\{[T] \in \overline{C V}_{N}:\left\langle T, \mu_{-}\right\rangle>\left\langle T, \mu_{+}\right\rangle\right\}
\end{aligned}
$$

Then the following hold:

(1) $V_{+}$is an open neighborhood of $\left[T_{+}\right]$and $V_{-}$is an open neighborhood of $\left[T_{-}\right]$in $\overline{C V}_{N}$ such that $V_{+} \varphi \subseteq V_{+}$and $V_{-} \varphi^{-1} \subseteq V_{-}$.

(2) $\cap_{n \geq 1} \bar{V}_{+} \varphi^{n}=\left\{\left[T_{+}\right]\right\}$and $\cap_{n \geq 1} \bar{V}_{-} \varphi^{-n}=\left\{\left[T_{-}\right]\right\}$.

(3) For any neighborhood $V$ of $\left[T_{+}\right]$there exists $n \geq 1$ such that $V_{+} \varphi^{n} \subseteq$ $V$ and for any neighborhood $V^{\prime}$ of $\left[T_{-}\right]$there is $n \geq 1$ such that $V_{-} \varphi^{-n} \subseteq V^{\prime}$.

(4) For any neighborhoods $V$ of $\left[T_{+}\right]$and $V^{\prime}$ of $\left[T_{-}\right]$in $\overline{C V}_{N}$ there exists $M \geq 1$ such that for every $n \geq M$ we have $\left(\overline{C V}_{N}-V^{\prime}\right) \varphi^{n} \subseteq V$ and $\left(\overline{C V}_{N}-V\right) \varphi^{-n} \subseteq V^{\prime}$

(5) The action of $\langle\varphi\rangle$ on $\overline{C V}_{N}-\left\{\left[T_{+}\right],\left[T_{-}\right]\right\}$is properly discontinuous and cocompact.

Remark 4.11. Note that Proposition 4.10 implies Proposition [3.4, and gives a new proof of the latter, using only the pointwise nature of the "NorthSouth" dynamics for hyperbolic iwips. This in turn also yields another proof of Corollary 3.5.

Lemma 4.12. Let $\varphi, \mu_{+}, \mu_{-}$be as in Convention 4.1, and let $A$ be a free basis of $F_{N}$. Then there exist an open set $U \subseteq \mathbb{P} C u r r\left(F_{N}\right)$ containing $\left[\mu_{+}\right]$ and an integer $M_{0} \geq 1$ with the following property:

For every $[\mu] \in U$ and every $n \geq M_{0}$ we have $\left\langle T_{A}, \varphi^{n} \mu\right\rangle \geq 2\left\langle T_{A}, \mu\right\rangle$.

Proof. Let $f: \Gamma \rightarrow \Gamma$ be a train-track representative of $\varphi$. There is an integer $L_{1} \geq 1$, an integer $M_{1} \geq 1$ and a constant $\lambda_{1}>1$ such that the following holds. If $\gamma=\gamma_{1} \gamma_{2} \gamma_{3}$ is a reduced concatenation of three reduced paths in $\Gamma$ where $\gamma_{2}$ is legal of simplicial length $\geq L_{1}$ then for any $n \geq M_{1}$ in the cancellation between the tightened forms of $f^{n} \gamma_{1}, f^{n} \gamma_{2}, f^{n} \gamma_{3}$, a segment of $f^{n} \gamma_{2}$ of simplicial length at least $\lambda_{1}\left|\gamma_{2}\right|$ survives.

Let $U$ be the neighborhood of $\left[\mu_{+}\right]$defined by the condition that for $[\mu] \in U$ the frequencies with respect to the simplicial metric on $\Gamma$ of all legal paths of simplicial length $L_{1}$ in $[\mu]$ are almost the same as they are for the corresponding frequencies in $\left[\mu_{+}\right]$and the frequencies of illegal turns are sufficiently close to zero. Here for a reduced path $v$ in $\Gamma$ and a nonzero current $\mu$ by a frequency of $v$ in $\mu$ we mean the ratio $\frac{\langle v, \mu\rangle_{\Gamma}}{\left\langle T_{\Gamma}, \mu\right\rangle}$. It is easy to see that this frequency depends only on $T_{\Gamma}$ and the projective class $[\mu]$ of $\mu$. See [31] for more details. Then for any rational current $\left[\eta_{w}\right] \in U$, where $w$ is a reduced cyclic path in $\Gamma$, when we write $w$ as a concatenation of maximal legal segments, the legal segments of length $\geq L_{1}$ each will constitute at least $1 / 2$ of the simplicial length of $w$. Then for any $k \geq 1$ there is $M \geq 1$ independent of $w$ such that $\left|\left[f^{n} w\right]\right| \geq 2^{k}|w|$ for every $w$ as above and every $n \geq M$. Therefore for every $[\mu] \in U$ we have $\left\langle T_{\Gamma}, \varphi^{n} \mu\right\rangle \geq 2^{k}\left\langle T_{\Gamma}, \mu\right\rangle$ for every 
$n \geq M$. Here $T_{\Gamma}$ is the universal cover of $\Gamma$ with the simplicial metric. Since the translation length functions on $F_{N}$ corresponding to the trees $T_{A}$ and on $T_{\Gamma}$ are bi-Lipschitz equivalent, it follows that, by choosing a sufficiently large $k$, there exists $M_{0} \geq 1$ such that for every $n \geq M_{0}$ and every $[\mu] \in U$ we have $\left\langle T_{A}, \varphi^{n} \mu\right\rangle \geq 2\left\langle T_{A}, \mu\right\rangle$, as required.

Corollary 4.13. Let $\varphi, \mu_{+}, \mu_{-}$be as in Convention 4.1, and let $A$ be a free basis of $F_{N}$.

Then for any neighborhood $V$ of $\left[\mu_{-}\right]$in $\mathbb{P} C u r r\left(F_{N}\right)$ there exists an integer $M_{1}=M_{1}(V, \varphi) \geq 1$ such that for every $[\mu] \in \mathbb{P} C u r r\left(F_{N}\right)-V$, for every $[T] \in \overline{C V}_{N}-U$ and any $n \geq M_{1}$ we have $\left\langle T_{A}, \varphi^{n} \mu\right\rangle \geq 2\left\langle T_{A}, \mu\right\rangle$.

Proof. Let $U$ and $M_{0}$ be provided by Lemma 4.12, By making $V$ smaller we may assume that $V$ is disjoint from $U$. By making $U$ smaller, via application of Lemma 4.5 and Lemma 4.6 we may assume that, in addition to the conditions guaranteed by Lemma 4.12, we have $\varphi(U) \subseteq U$. Note that since $\varphi(U) \subseteq U$, then for every $[\nu] \in U$ and every integer $k \geq 1$ we have $\left\langle T_{A}, \varphi^{k M_{0}} \nu\right\rangle \geq 2^{k}\left\langle T_{A}, \nu\right\rangle$.

By Lemma 4.7 there exists $M \geq 1$ such that $\varphi^{n}\left(\mathbb{P C u r r}\left(F_{N}\right)-V\right) \subseteq U$ for every $n \geq M$. Since $M, M_{0}$ are fixed, there exists $C>0$ such that for every $\mu \in \operatorname{Curr}\left(F_{N}\right)$ we have $\left\langle T_{A}, \varphi^{M} \mu\right\rangle \geq C\left\langle T_{A}, \mu\right\rangle$. Let $k \geq 1$ be an arbitrary integer. Let $[\mu] \in \mathbb{P} \operatorname{Curr}\left(F_{N}\right)-V$ be arbitrary. We have $\varphi^{n}([\mu]) \in U$ for every $n \geq M$. We also have $\left\langle T_{A}, \varphi^{M} \mu\right\rangle \geq C\left\langle T_{A}, \mu\right\rangle$ and $\left\langle T_{A}, \varphi^{k M_{0}+M} \mu\right\rangle \geq$ $2^{k}\left\langle T_{A}, \varphi^{M} \mu\right\rangle$ by Lemma 4.12, Therefore $\left\langle T_{A}, \varphi^{k M_{0}+M} \mu\right\rangle \geq 2{ }^{k} C\left\langle T_{A}, \mu\right\rangle$. The statement of the corollary now easily follows.

Corollary 4.14. Let $\varphi, \psi \in \operatorname{Out}\left(F_{N}\right)$ be hyperbolic iwips such that $\langle\varphi, \psi\rangle$ is not virtually cyclic. Then there exists $M \geq 1$ such that for any $n, m \geq M$ the subgroup $\left\langle\varphi^{n}, \psi^{m}\right\rangle$ is free of rank two.

Proof. Corollary 3.11 implies that $\left[T_{ \pm}(\varphi)\right]$ and $\left[T_{ \pm}(\psi)\right]$ are four distinct points of $\overline{\mathrm{CV}}_{N}$. Therefore the statement follows from Corollary 3.5 ,

Note that by Proposition 3.7 for hyperbolic iwips $\varphi, \psi$ the assumption that $\left[T_{ \pm}(\varphi)\right],\left[T_{ \pm}(\psi)\right]$ are four distinct points of $\overline{\mathrm{CV}}_{N}$ is equivalent to the condition that $\left[\mu_{ \pm}(\varphi)\right],\left[\mu_{ \pm}(\psi)\right]$ are four distinct points of $\mathbb{P} \operatorname{Curr}\left(F_{N}\right)$.

Lemma 4.15. Let $\varphi, \psi \in$ Out $\left(F_{N}\right)$ be hyperbolic iwips such that $\langle\varphi, \psi\rangle \subseteq$ $\operatorname{Out}\left(F_{N}\right)$ is not virtually cyclic. Let $A$ be a free basis of $F_{N}$. Then there exists $M \geq 1$ with the following property:

For any $\mu \in \operatorname{Curr}\left(F_{N}\right)$ and for any $n, m \geq M$, for at least three out of four elements $\alpha$ of $\left\{\varphi^{n}, \psi^{m}, \varphi^{-n}, \psi^{-m}\right\}$ we have

$$
2\left\langle T_{A}, \mu\right\rangle \leq\left\langle T_{A}, \alpha \mu\right\rangle \text {. }
$$

Proof. Since $\langle\varphi, \psi\rangle \subseteq \operatorname{Out}\left(F_{N}\right)$ is not virtually cyclic, the four eigencurrents $\left[\mu_{+}(\varphi)\right],\left[\mu_{+}(\psi)\right],\left[\mu_{-}(\varphi)\right],\left[\mu_{-}(\psi)\right]$ are four distinct elements in $\mathbb{P C u r r}\left(F_{N}\right)$. Take four disjoint open neighborhoods $U_{+}(\varphi), U_{+}(\psi), U_{-}(\varphi), U_{-}(\psi)$. 
Let $M \geq 1$ be the maximum of the the constants $M_{1}\left(U_{-}(\varphi), \varphi\right), M_{1}\left(U_{-}(\psi), \psi\right)$, $M_{1}\left(U_{+}(\varphi), \varphi^{-1}\right), M_{1}\left(U_{+}(\psi), \psi^{-1}\right)$ provided by Corollary 4.13, Let $\mu \in$ $\operatorname{Curr}\left(F_{N}\right), \mu \neq 0$ be arbitrary. Then there are at least three of the four sets $U_{+}(\varphi), U_{+}(\psi), U_{-}(\varphi), U_{-}(\psi)$ that $[\mu]$ does not belong to, and the statement of the lemma follows from Corollary 4.13

Lemma 4.15 immediately implies:

Corollary 4.16. Let $\varphi, \psi \in \operatorname{Out}\left(F_{N}\right)$ be hyperbolic iwips such that $\langle\varphi, \psi\rangle \subseteq$ $\operatorname{Out}\left(F_{N}\right)$ is not virtually cyclic. Let $A$ be a free basis of $F_{N}$. Then there exists $M \geq 1$ with the following property: For any $w \in F_{N}$ and any $n, m \geq M$, for at least three out of four elements $\alpha$ of $\left\{\varphi^{n}, \psi^{m}, \varphi^{-n}, \psi^{-m}\right\}$ we have

$$
2\|w\|_{A} \leq\|\alpha(w)\|_{A}
$$

Via a standard argument (c.f. the proof of Theorem 5.2 in [5]), Corollary 4.16 and the Bestvina-Feighn Combination Theorem [1] imply:

Theorem 4.17. Let $N \geq 3$ and let $\varphi, \psi \in \operatorname{Out}\left(F_{N}\right)$ be hyperbolic iwips such that $\langle\varphi, \psi\rangle \subseteq \operatorname{Out}\left(F_{N}\right)$ is not virtually cyclic and let $\Phi, \Psi \in \operatorname{Aut}\left(F_{N}\right)$ be such that $\Phi$ represents $\varphi$ and $\Psi$ represents $\psi$. There exists $M \geq 1$ such that for any $n, m \geq M$ the subgroup $\left\langle\varphi^{n}, \psi^{m}\right\rangle \subseteq \operatorname{Out}\left(F_{N}\right)$ is free of rank two, every nontrivial element of this subgroup is hyperbolic and the group

$$
\left.G_{n, m}=\left\langle F_{N}, t, s\right| t^{-1} w t=\Phi^{n}(w), s^{-1} w s=\Psi^{m}(w) \text { for every } w \in F_{N}\right\rangle
$$

is word-hyperbolic.

The "3 out of 4" condition in Corollary 4.16 was first introduced by Lee Mosher for surface homeomorphisms in [46] where he used it to construct an example of a (closed surface)-by-(free of rank two) word-hyperbolic group. Similarly, the "3 out of 4" condition was used by Bestvina, Feign and Handel [5] to construct a free-by-free word-hyperbolic group. Our proof of the "3 out of 4" condition in Corollary 4.16 is different from both the approaches of Mosher and of Bestvina-Feighn-Handel: our method is based on exploiting North-South dynamics of hyperbolic iwips acting on the space of projectivized currents rather than on the space of laminations.

Remark 4.18. In Theorem 4.17 it is also easy to conclude that for every nontrivial element $\theta \in\langle\Phi, \Psi\rangle \subseteq \operatorname{Aut}\left(F_{N}\right)$ the automorphism $\theta$ is hyperbolic. This can be seen directly from the Annuli Flare Condition [1] for the group $G_{n, m}$ above. Alternatively, suppose $\theta$ is not hyperbolic. Then $\theta$ is not atoroidal, that is to say $\theta$ has a periodic conjugacy class. This yields a $\mathbb{Z} \times \mathbb{Z}$ subgroup in $G_{n i, m}$ which contradicts the fact that $G_{n, m}$ is word-hyperbolic.

Remark 4.19. The same proof as that of Theorem 4.17 shows that the conclusion of this theorem holds if instead of two elements of $\operatorname{Out}\left(F_{N}\right)$ we use $k \geq 2$ hyperbolic iwip elements $\varphi_{1}, \ldots, \varphi_{k}$ with the property that for every $1 \leq i<j \leq k$ the subgroup $\left\langle\varphi_{i}, \varphi_{j}\right\rangle \subseteq \operatorname{Out}\left(F_{N}\right)$ is not virtually cyclic. 


\section{Ping-Pong For Schottky type Groups And its CONSEquences}

Definition 5.1. Let $G$ be a group that acts on a non-empty set $X$ (either on the left or on the right). Suppose that the group $G$ is generated by two specified elements $g$ and $h$, and that $X$ contains pairwise disjoint geographical nonempty subsets $N$ (= "North"), $S$ (= "South"), $E$ (= "East") an $W$ (= "West"), such that $g$ maps $X-S$ into $N, g^{-1}$ maps $X-N$ into $S$, and similarly $h$ with $E$ and $W$. We call this action of $G$ on $X$ a 2-generator ping-pong action with respect to $g$ and $h$.

For the rest of this section, unless specified otherwise we assume that we are given a 2-generator ping-pong action of $G$ on $X$ with respect to $g$ and $h$.

It follows from Felix Klein's classical argument that in the above situation $G$ is free with a free basis $\{g, h\}$. Notice that we purposefully did not specify whether $G$ acts from the left or from the right on $X$. For any reduced and cyclically reduced word $w=x_{1} \ldots x_{q}$ in $\{g, h\}^{ \pm 1}$ we define the final acting letter to be the $x_{i}$ that acts last on $X$. Thus, if we have a left action, then the final acting letter of $w$ is the first letter $x_{1}$, and in case of a right action it is the last letter $x_{q}$.

We define the forward limit region of $w$ (reduced and cyclically reduced) as the nested intersections of the images of $Y$ under $w^{n}$, for positive $n$, where $Y=N$ if $w$ has the final acting letter $g, Y=S$ if $w$ has the final acting letter $g^{-1}$, and similarly $Y=E$ or $Y=W$ if $w$ has the final acting letter $h$ or $h^{-1}$.

Remark 5.2. It follows directly from the above set-up that for a cyclically reduced $w$, any fixed point of $w$ in $X$ must be contained either in the forward limit region of $w$ or in that of $w^{-1}$ (one could call the latter the backward limit region of $w)$.

Definition 5.3. A 2-generator ping-pong action of $G$ on $X$ as above is called open if in addition $X$ is a topological space, $G$ acts by homeomorphisms on $X$, and the geographical subsets $N, S, E$ and $W$ are open.

Notice also that the restriction of any 2-generator ping-pong action to a $G$-invariant subset $X^{\prime} \subseteq X$ is also 2-generator ping-pong: One simply redefines the set North as $X^{\prime} \cap N$, South as $X^{\prime} \cap S$, etc. Of course, if the action on $X$ is open, then so is the action on $X^{\prime}$.

Suppose now that $\varphi, \psi \in \operatorname{Out}\left(F_{N}\right)$ are hyperbolic iwips such that $\langle\varphi, \psi\rangle$ is not virtually cyclic. We already know by the results of Section 4 that there is $M \geq 1$ such that for every $n, m \geq M$ the actions of $G=\left\langle\varphi^{n}, \psi^{m}\right\rangle$ on both, $\overline{\mathrm{CV}}_{N}$ (from the right) and on $\mathbb{P C u r r}\left(F_{N}\right)$ (from the left) are open 2 -generator ping-pong actions with respect to $\varphi^{n}, \psi^{m}$. Note that in this case for the left action of $G$ on $\mathbb{P} \operatorname{Curr}\left(F_{N}\right)$, the "north" set $N$ contains $\left[\mu_{+}(\varphi)\right]$, the "south" set $S$ contains $\left[\mu_{-}(\varphi)\right]$, the "east" set $E$ contains $\left[\mu_{+}(\psi)\right]$ and the "west" set $W$ contains $\left[\mu_{-}(\psi)\right]$. Similarly, for the right action of $G$ on 
$\overline{\mathrm{CV}}_{N}$ the geographical sets $N, S, E, W$ contain $\left[T_{+}(\varphi)\right],\left[T_{-}(\varphi)\right],\left[T_{+}(\psi)\right]$, and $\left[T_{-}(\psi)\right]$ respectively.

The main goal of this section is to prove the following:

Proposition 5.4. Let $\varphi, \psi \in \operatorname{Out}\left(F_{N}\right)$ be hyperbolic iwips, and assume that the actions of $G=\langle\varphi, \psi\rangle$ on both, $\overline{C V}_{N}$ (from the right) and on $\mathbb{P} C u r r\left(F_{N}\right)$ (from the left) are open 2-generator ping-pong actions with respect to $\varphi, \psi$.

Then there exist constants $m_{0}, n_{0} \geq 1$ with the following property. Suppose $m \geq m_{0}, n \geq n_{0}$ and $w$ is a cyclically reduced word in $\varphi^{ \pm 1}, \psi^{ \pm 1}$ which starts in $\varphi^{m}$ and ends in $\varphi^{n}$, and suppose $[\mu] \in \mathbb{P} C u r r\left(F_{N}\right)$ is such that $[\mu]$ is contained in the forward limit region of $w$ and such for some $\lambda \neq 1$ we have $w \mu=\lambda \mu$.

Then $\lambda>1$.

For the remainder of this section we suppose that the assumptions of Proposition 5.4 are satisfied. In order to prove this proposition we need first some preliminary considerations.

Recall that $\mathrm{CV}_{N}=\mathbb{P c v}_{N}$ is the projectivization of $\mathrm{cv}_{N}$. We consider the (right) action of $G$ on $\overline{\mathrm{CV}}_{N}$. Denote by $c v_{N}^{1} \subseteq \mathrm{cv}_{N}$ the lift of $\mathrm{CV}_{N}$ to $\operatorname{cv}_{N}$ where every tree has covolume 1 . Note that $c v_{N}^{1}$ is invariant under the action of $\operatorname{Out}\left(F_{N}\right)$ on $\mathrm{cv}_{N}$, and that the group $G=\langle\varphi, \psi\rangle$ acts on $c v_{N}^{1}$ again as open 2-generator ping-pong group, since by the hypothesis of Proposition 5.4 it does so on $\overline{\mathrm{CV}}_{N}$ and thus on $\mathrm{CV}_{N}$.

Convention 5.5. Let $\varphi \in \operatorname{Out}\left(F_{N}\right)$ be a hyperbolic iwip. Let $\left[\mu_{+}\right]=$ $\left[\mu_{+}(\varphi)\right]$ be an expanding fixed projectivized current of $\varphi$, i.e. $\varphi \mu_{+}=\lambda_{+} \mu_{+}$, with $\lambda_{+}>1$. Let $\left[T_{-}\right]$be a contracting fixed projectivized $\mathbb{R}$-tree of $\varphi$, i.e. $T_{-} \varphi=\lambda_{-}^{-1} T_{-}$, with $\lambda_{-}>1$. We recall that, by Proposition 3.2 and Proposition 3.3, both $\mu_{+}$and $T_{-}$are uniquely determined by $\varphi$ up to scalar multiplication, and that $\left\langle T_{-}, \mu_{+}\right\rangle=0$. Moreover, by Proposition 3.7, up to scalar multiples, $T_{-}$is the unique tree $T \in \overline{\mathrm{cv}}_{N}$ satisfying $\left\langle T, \mu_{+}\right\rangle=0$.

Similarly, let $\left[\mu_{-}\right]=\left[\mu_{-}(\varphi)\right]$ and $\left[T_{+}\right]=\left[T_{+}(\varphi)\right]$ be the contracting fixed projectivized current and expanding fixed projectivized tree for $\varphi$. Note that $T_{+} \varphi=\lambda_{+} T_{+}$and $\varphi^{-1} \mu_{-}=\lambda_{-} \mu_{-}$.

For any subset $V \subseteq \overline{\mathrm{cv}}_{N}$ we denote by $\mathbb{P} V \subseteq \overline{\mathrm{CV}}_{N}$ its canonical image under projectivization. Note that the closure $\overline{\bar{V}}$ of $V$ in $\overline{\mathrm{cv}}_{N}$ projects to a subset $\mathbb{P} \bar{V} \subseteq \overline{\mathrm{CV}}_{N}$ which is contained in the closure $\overline{\mathbb{P} V}$ of $\mathbb{P} V$ in $\overline{\mathrm{CV}}_{N}$, but that in general the two are not equal:

$$
\mathbb{P} \bar{V} \subseteq \overline{\mathbb{P} V} .
$$

Lemma 5.6. Let $V$ be a subset of $c v_{N}^{1}$ with the property that $\left[T_{-}\right] \notin \overline{\mathbb{P} V}$. Suppose also that for some constant $c>0$ we have

$$
\left\langle T, \mu_{+}\right\rangle \geq c
$$


for all $T$ in $V$. Then for some sufficiently small neighborhood $U \subseteq \operatorname{Curr}\left(F_{N}\right)$ of $\mu_{+}$one has

$$
\langle T, \mu\rangle \geq \frac{c}{2}
$$

for any $T$ in $V$ and for any $\mu \in U$.

Proof. Suppose that the statement of the lemma is false. Then there exist a sequence of currents $\mu_{i} \in \operatorname{Curr}\left(F_{N}\right)$ converging to $\mu_{+}$, and a sequence of trees $T_{i} \in V$, which all satisfy

$$
\left\langle T_{i}, \mu_{i}\right\rangle<\frac{c}{2} \leq \frac{\left\langle T_{i}, \mu_{+}\right\rangle}{2} .
$$

By compactness of $\overline{\mathrm{CV}}_{N}$ we can extract a subsequence of the $T_{i}$, which we still denote $T_{i}$, which converges projectively to some $\mathbb{R}$-tree $T_{\infty}$ in $\overline{\mathrm{cv}}_{N}$. Let $\lambda_{i}>0$ be such that $\lim _{i \rightarrow \infty} \lambda_{i} T_{i}=T_{\infty}$ in $\overline{\mathrm{cv}}_{N}$.

By continuity and linearity of the intersection form we have:

$$
\begin{aligned}
& \left\langle T_{\infty}, \mu_{+}\right\rangle=\left\langle\lim \lambda_{i} T_{i}, \lim \mu_{i}\right\rangle=\lim \lambda_{i}\left\langle T_{i}, \mu_{i}\right\rangle \\
\leq & \lim \frac{\lambda_{i}\left\langle T_{i}, \mu_{+}\right\rangle}{2}=\frac{\left\langle\lim \lambda_{i} T_{i}, \mu_{+}\right\rangle}{2}=\frac{\left\langle T_{\infty}, \mu_{+}\right\rangle}{2}
\end{aligned}
$$

Hence $\left\langle T_{\infty}, \mu_{+}\right\rangle=0$ and therefore, by Proposition [3.7, $\left[T_{\infty}\right]=\left[T_{-}\right]$. However, $\left[T_{\infty}\right]$ is contained in the closure of $\mathbb{P} V$ in $\overline{\mathrm{CV}}_{N}$, which contradicts our assumption that $\left[T_{-}\right] \notin \overline{\mathbb{P} V}$.

Lemma 5.7. There exists a non-empty subset $V \subseteq c v_{N}^{1}$ with the following properties:

(1) $\left[T_{-}\right] \notin \overline{\mathbb{P} V}$

(2) $c:=\inf \left\{\left\langle T, \mu_{+}\right\rangle \mid T \in V\right\}>0$

(3) $V \varphi \subseteq V$, and $\left\langle T \varphi, \mu_{+}\right\rangle=\lambda_{+}\left\langle T, \mu_{+}\right\rangle$for any $T \in V$.

(4) There exists a tree $T_{0} \in V$ such that for every reduced word $w$ in $\varphi^{ \pm 1}$ and $\psi^{ \pm 1}$ that does not end in $\varphi^{-1}$, the tree $T_{0} w$ lies in $V$.

Proof. Let $N, S, E, W \subseteq \overline{\mathrm{CV}}_{N}$ be the geographical subsets for the ping-pong action of $G=\langle\varphi, \psi\rangle$ on $\overline{\mathrm{CV}}_{N}$. Recall that $N$ and $S$ are open neighborhoods of $\left[T_{+}\right]=\left[T_{+}(\varphi)\right]$ and $\left[T_{-}\right]=\left[T_{-}(\varphi)\right]$ accordingly. Choose an arbitrary $T_{0} \in c v_{N}^{1}$ such that $\left[T_{0}\right] \notin N \cup S \cup E \cup W$.

Put

$$
V=\left\{T \in T_{0} \operatorname{Out}\left(F_{N}\right):[T] \notin S\right\} .
$$

We claim that these choices of $V$ and $T_{0}$ satisfy all the requirements of the lemma. Indeed, conditions (1), (3) and (4) follow immediately from the definitions of $V$ and $T_{0}$ and from the ping-pong properties of the action of $G$ on $\overline{\mathrm{CV}}_{N}$. Suppose that condition (2) fails. Then there exists a sequence $\alpha_{n} \in \operatorname{Out}\left(F_{N}\right)$ such that $\lim _{n \rightarrow \infty}\left\langle T_{0} \alpha_{n}, \mu_{+}\right\rangle=0$. After passing to a further subsequence, we may assume that $\lim _{n \rightarrow \infty}\left[T_{0} \alpha_{n}\right]=\left[T_{\infty}\right]$ and that $\lim _{n \rightarrow \infty} c_{n} T_{0} \alpha_{n}=T_{\infty}$ for some $T_{\infty} \in \overline{\mathrm{cv}}_{N}$ and some $c_{n}>0$. Note that $\left[T_{\infty}\right]$ belongs to the closure of $\mathbb{P} V$ and hence $\left[T_{\infty}\right] \neq\left[T_{-}\right]$. Note that because 
$T_{0} \in c v_{N}^{1}$, we have $\left\langle T_{0} \alpha_{n}, \mu_{+}\right\rangle>0$. After passing to a further subsequence we may also assume that all $\alpha_{n}$ are distinct and that

$$
0<\left\langle T_{0} \alpha_{n}, \mu_{+}\right\rangle \leq 1
$$

for all $n \geq 1$. Since $\alpha_{n}$ are distinct and the action of $\operatorname{Out}\left(F_{N}\right)$ on $c v_{N}^{1}$ is properly discontinuous, it follows that $\left[T_{\infty}\right] \in \partial \mathrm{CV}_{N}=\overline{\mathrm{CV}}_{N}-\mathrm{CV}_{N}$. This in turn implies that $\lim _{n \rightarrow \infty} c_{n}=0$ (see [35] for details).

By the linearity of the intersection form we have:

$$
\left\langle c_{n} T_{0} \alpha_{n}, \mu_{+}\right\rangle=c_{n}\left\langle T_{0} \alpha_{n}, \mu_{+}\right\rangle \leq c_{n} \rightarrow_{n \rightarrow \infty} 0
$$

Hence, by continuity, $\left\langle T_{\infty}, \mu_{+}\right\rangle=0$ which, by Proposition 3.7, implies that $\left[T_{\infty}\right]=\left[T_{-}\right]$. This contradicts our earlier conclusion that $\left[T_{\infty}\right] \neq\left[T_{-}\right]$.

Lemma 5.8. There exists a tree $T_{0} \in c v_{N}^{1}$, a constant $n_{0} \geq 0$ and a neighborhood $U \subseteq \operatorname{Curr}\left(F_{N}\right)$ of $\mu_{+}$, such that for any reduced word $w$ in $\varphi^{ \pm 1}$ and $\psi^{ \pm 1}$ that does not end in $\varphi^{-1}$, for any $n \geq n_{0}$, and for any $\mu \in U$, one has:

$$
\left\langle T_{0}, w \varphi^{n} \mu\right\rangle>\left\langle T_{0}, \mu\right\rangle \text {. }
$$

Proof. Consider a set $V \subseteq c v_{N}^{1}$ and a tree $T_{0} \in V$ as in Lemma 5.7. By definition we have $\lambda_{+}>1$, and by Lemma $5.7(2)$, the infimum $c$ of all $\left\langle T, \mu_{+}\right\rangle$, for any $T \in V$, satisfies $c>0$. Thus we can pick $n_{0} \geq 0$ so that $\lambda_{+}^{n_{0}} c>100\left\langle T_{0}, \mu_{+}\right\rangle$. From the continuity of the intersection form we deduce

$$
\frac{\lambda_{+}^{n} c}{2}>\left\langle T_{0}, \mu\right\rangle
$$

for all $n \geq n_{0}$ and for any $\mu$ in some sufficiently small neighborhood $U_{0}$ of $\mu_{+}$.

By part (4) of Lemma 5.7 the tree $T_{0} w$ is contained in $V$, and hence $T_{0} w \varphi^{n}$ is contained in $V \varphi^{n}$. From parts (2) and (3) of Lemma 5.7 we deduce that every $T \in V \varphi^{n}$ satisfies:

$$
\left\langle T, \mu_{+}\right\rangle \geq \lambda_{+}^{n} c
$$

Now Lemma 5.6, whose hypotheses are guaranteed by Lemma 5.7 (1) and (2), implies that

$$
\langle T, \mu\rangle \geq \frac{\lambda_{+}^{n} c}{2}
$$

for any $T \in V \varphi^{n}$ and any $\mu$ in a sufficiently small neighborhood $U^{\prime} \subseteq$ $\operatorname{Curr}\left(F_{N}\right)$ of $\mu_{+}$. It follows from our above choice of $n_{0}$ that

$$
\left\langle T_{0}, w \varphi^{n} \mu\right\rangle=\left\langle T_{0} w \varphi^{n}, \mu\right\rangle \geq \frac{\lambda_{+}^{n} c}{2}>\left\langle T_{0}, \mu\right\rangle
$$

for any $\mu$ in $U:=U^{\prime} \cap U_{0}$.

We now consider the (left) action of $G$ on $\mathbb{P C u r r}\left(F_{N}\right)$, which by the hypotheses of Proposition 5.4 is again an open 2-generator ping-pong action.

By the North-South dynamics of iwips (see the results of Section 4) for any sufficiently large $m \geq 1$ the power $\varphi^{m}$ maps any compact subset of $\mathbb{P} \operatorname{Curr}\left(F_{N}\right)$, that excludes the repelling fixed point $\left[\mu_{-}\right]$of $\varphi$, into $\mathbb{P} U \subseteq$ 
$\mathbb{P} \operatorname{Curr}\left(F_{N}\right)$. Here $\mathbb{P} U \subseteq \mathbb{P} \operatorname{Curr}\left(F_{N}\right)$ means the image of a neighborhood $U \subseteq \operatorname{Curr}\left(F_{N}\right)$ of the $\varphi$-expanding eigencurrent $\mu_{+}$as above. As an easy consequence of the open 2-generator ping-pong assumption of the $G$-action on $\mathbb{P} \operatorname{Curr}\left(F_{N}\right)$ we thus obtain:

Lemma 5.9. For every neighborhood $U \subseteq \operatorname{Curr}\left(F_{N}\right)$ of $\mu_{+}$there exists $m_{1} \geq 1$ such that for all $m \geq m_{1}$ and for every reduced word $w$ in $\varphi^{ \pm 1}$ and $\psi^{ \pm 1}$ which does not start or end with $\varphi^{-1}$, the forward limit region of $\varphi^{m} w$ in $\mathbb{P} \operatorname{Curr}\left(F_{N}\right)$ is contained in $\mathbb{P} U$.

We can now prove Proposition 5.4:

Proof of Proposition 5.4. Let $U, T_{0}, n_{0}$ and $m_{1}$ be as given in Lemma 5.8 and Lemma 5.9, so that both apply to the given word $w=\varphi^{m} w^{\prime} \varphi^{n}$, where $w^{\prime}$ is a reduced word in $\varphi^{ \pm 1}$ and $\psi^{ \pm 1}$ which does not start or end with $\varphi^{-1}$, and where $n \geq n_{0}$ and $m \geq m_{0}:=\max \left(m_{1}, n_{0}\right)$ holds.

Recall that by assumptions of Proposition 5.4, we are given a projective current $[\mu]$ in the forward limit region of $w$ such that $[\mu]$ is fixed by $w$, so that $w \mu=\lambda \mu$ for some $\lambda>0$. We need to prove that $\lambda>1$.

Since $[\mu]$ contained in the forward limit region of $w$ in $\mathbb{P C u r r}\left(F_{N}\right)$, it follows that $[\mu]$ is contained in the set $\mathbb{P} U$, by Lemma 5.9. Hence some scalar multiple $\mu^{\prime}$ of $\mu$ is contained in $U$, and we deduce from Lemma 5.8 that:

$$
\left\langle T_{0}, w \mu^{\prime}\right\rangle=\left\langle T_{0}, \varphi^{m} w^{\prime} \varphi^{n} \mu^{\prime}\right\rangle>\left\langle T_{0}, \mu^{\prime}\right\rangle
$$

But since $\mu$ is projectively fixed by $w=\varphi^{m} w^{\prime} \varphi^{n}$, we have:

$$
\left\langle T_{0}, w \mu\right\rangle=\left\langle T_{0}, \lambda \mu\right\rangle=\lambda\left\langle T_{0}, \mu\right\rangle
$$

Hence $\lambda\left\langle T_{0}, \mu\right\rangle>\left\langle T_{0}, \mu\right\rangle$. Note that $T_{0}$ is a tree with a free simplicial action of $F_{N}$, which implies $\left\langle T_{0}, \mu\right\rangle \neq 0$. Therefore

$$
\lambda>1 \text {, }
$$

which proves Proposition 5.4 .

An interesting feature of the above proof is that it needs the ping-pong property of the action of $G$ on both spaces, $\overline{\mathrm{CV}}_{N}$ and $\mathbb{P} \operatorname{Curr}\left(F_{N}\right)$. As one of these actions is a left action and the other one a right action, the words $w$ considered must both, start and end in large powers of $\varphi$. However, this is not a problem for the application in the next section, since the property of an automorphism to be an iwip is invariant under conjugation in $\operatorname{Out}\left(F_{N}\right)$.

Recall that $\varphi, \mu_{+}=\mu_{+}(\varphi)$ and $\mu_{-}=\mu_{-}(\varphi)$ are as in Convention 5.5. We finish this section with a lemma that will be used in the next section:

Lemma 5.10. There exists neighborhoods $U_{+}(\varphi) \subseteq \operatorname{Curr}\left(F_{N}\right)$ of $\mu_{+}$and $U_{-}(\varphi) \subseteq \operatorname{Curr}\left(F_{N}\right)$ of $\mu_{-}$such that

$$
\left\langle T, \mu_{+}^{\prime}\right\rangle+\left\langle T, \mu_{-}^{\prime}\right\rangle>0
$$

for any $T \in \overline{c v}_{N}$ and any $\mu_{+}^{\prime} \in U_{+}, \mu_{-}^{\prime} \in U_{-}$. 
Proof. It suffices to recall that, by Proposition 3.7, the above inequality is true for $\mu_{+}^{\prime}=\mu_{+}$and $\mu_{-}^{\prime}=\mu_{-}$, and to use the continuity of the intersection form.

\section{EVERY SCHOtTKY GROUP CONTAINS A RANK-TWO FREE IWIP SUBGROUP}

We first prove a property that characterizes those hyperbolic automorphisms of $F_{N}$ which are not iwips.

Proposition 6.1. For every $\Phi \in \operatorname{Aut}\left(F_{N}\right)$ which is hyperbolic but not an iwip there exist $k \geq 1$, a tree $T_{0} \in \overline{c v}_{N}$ and currents $\nu_{+}, \nu_{-} \in \operatorname{Curr}\left(F_{N}\right) \backslash\{0\}$ with the following properties:

(1) $\Phi^{k}\left(\nu_{+}\right)=\rho_{+} \nu_{+}$and $\Phi^{k}\left(\nu_{-}\right)=\rho_{-}^{-1} \nu_{-}$, for some $\rho_{+}, \rho_{-}>1$.

(2) $\left\langle T_{0}, \nu_{+}\right\rangle=\left\langle T_{0}, \nu_{-}\right\rangle=0$.

Proof. The $\mathbb{R}$-tree $T_{0}$ is constructed as described in [21] from a relative train track representative (in the sense of [3]) $f: \tau \rightarrow \tau$ of $\Phi$ using a (row) eigenvector of the transition matrix $M(f)$ which has non-zero coefficients only for the top stratum of $\tau$. The translation length with respect to $T_{0}$ satisfies

$$
\|w\|_{T_{0}}=0
$$

for all conjugacy classes $[w] \subseteq F_{N}$ which are represented by a loop in $\tau$ that does not traverse any edge of the top stratum. Note that $T_{0}$ is projectively $\Phi$-invariant, but that the stretching factor may well be equal to 1 , in which case $T_{0}$ is simplicial.

Since $\Phi$ has a reducible power, we can choose a proper free factor $U$ of $F_{N}$ which is (up to conjugation) fixed by some power $\Phi^{k}$ with $k \geq 1$, and which does not contain properly any non-trivial free factor of $F_{N}$ that is $\Phi^{h}$-invariant (up to conjugation) for any $h \geq 1$. After composing $\Psi=\Phi^{k}$ with an inner automorphism if necessary, we may assume that $\Psi(U)=U$. Note that $U$ is not cyclic since by assumption $\Phi$ is hyperbolic.

We claim that, moreover, $U$ can be chosen in such a way that $U$ fixes a point of $T_{0}$. Indeed, since every maximal elliptic subgroup for the above tree $T_{0}$ is (up to conjugation) a $\Phi$-invariant free factor of $F_{N}$, if such a subgroup is non-trivial, it must contain the $\Phi$-orbit of some free factor $U$ as above. It thus remains to argue that there exist at least one elliptic subgroup of $T_{0}$ which is non-trivial. If $T_{0}$ is simplicial, this is obvious, as otherwise the action of $F_{N}$ on $T_{0}$ would be free, and since $T_{0}$ is $\Phi$-invariant, the automorphism $\Phi$ would be periodic (up to conjugation) and hence not hyperbolic. In the complementary case, where $T_{0}$ is not simplicial, the top stratum of the above train track map $f: \tau \rightarrow \tau$ must be exponentially growing. Thus, if $\tau$ has more than one stratum, every lower stratum contributes to the elliptic subgroups of $T_{0}$, and hence the bottom stratum would define a non-trivial elliptic subgroup for $T_{0}$, as required. Finally, if there is only one stratum in $\tau$, then either $\Phi$ was an iwip, or else, by Proposition 5.1 of [29] (see also 
chapter 7 of [42]), one of the vertices of $\tau$ can be blown-up to give a new $\Phi$ invariant train track with a periodic top stratum. For this new train track $\tau^{\prime}$ its stable tree $T_{0}^{\prime}$ will be simplicial (as explained in detail in the proof of Proposition 5.1 of [29]), and the previous arguments would apply. Thus indeed $U$ and $T_{0}$ can be chosen so that $U$ fixes a point in $T_{0}$.

By the choice of $U$, the restriction $\left.\Psi\right|_{U} \in A u t(U)$ is an iwip automophism of $U$. Moreover, $\left.\Psi\right|_{U}$ has no periodic conjugacy classes (since $\Phi$ has no periodic conjugacy classes), so that $\left.\Psi\right|_{U} \in \operatorname{Aut}(U)$ is also atoroidal.

Therefore there exist a projectively unique $\left.\Psi\right|_{U}$-invariant expanding current $\mu_{+}^{U}$ and a projectively unique $\left.\Psi\right|_{U^{-}}$invariant contracting current $\mu_{-}^{U}$ in $\operatorname{Curr}(U)$. More precisely, $\left.\Psi\right|_{U}\left(\mu_{+}^{U}\right)=\rho_{+} \mu_{+}^{U}$ and $\left(\left.\Psi\right|_{U}\right)^{-1}\left(\mu_{-}^{U}\right)=\rho_{-} \mu_{-}^{U}$ for some $\rho_{+}, \rho_{-}>1$.

Recall that, as shown in [31, the inclusion $\iota: U \rightarrow F_{N}$ defines a continuous linear map $\iota_{*}: \operatorname{Curr}(U) \rightarrow \operatorname{Curr}\left(F_{N}\right)$ which extends the obvious map on conjugacy classes. Namely, for any nontrivial $u \in U$ we have $\iota_{*}\left(\eta_{u}^{U}\right)=\eta_{u}^{F_{N}}$ where $\eta_{u}^{U} \in \operatorname{Curr}(U)$ and $\eta_{u}^{F_{N}} \in \operatorname{Curr}\left(F_{N}\right)$ are the rational currents defined by $u$ on $U$ and $F_{N}$ respectively. Moreover, the map $\iota_{*}$ has a particularly simple form in a simplicial chart corresponding to a free basis $A$ of $F_{N}$ of the form $A=B \sqcup C$ where $B$ is a free basis of $U$. Namely, if we use $A$ as a simplicial chart on $F_{N}$ and $B$ as a simplicial chart on $U$ then for every $\mu \in \operatorname{Curr}(U)$ and every nontrivial freely reduced word $v \in F(A)$ we have:

$$
\left\langle v, \iota_{*} \mu\right\rangle_{A}=\left\{\begin{array}{l}
\langle v, \mu\rangle_{B}, \quad \text { if } v \in F(B)=U \\
0, \quad \text { otherwise. }
\end{array}\right.
$$

Put $\nu_{+}=\iota_{*}\left(\mu_{+}^{U}\right)$ and $\nu_{-}=\iota_{*}\left(\mu_{-}^{U}\right)$.

By the main result of [35], since the supports of $\nu_{+}$and $\nu_{-}$are carried by $U$ and since every element of $U$ has translation length zero on $T_{0}$, it follows that $\left\langle T_{0}, \nu_{+}\right\rangle=\left\langle T_{0}, \nu_{-}\right\rangle=0$.

We claim that $\Psi \nu_{+}=\rho_{+} \nu_{+}$and $\Psi \nu_{-}=\rho_{-}^{-1} \nu_{-}$. Indeed, choose a nontrivial element $a \in U$. We know that (up to rescaling $\nu_{+}$),

$$
\mu_{+}^{U}=\lim _{n \rightarrow \infty} \frac{\left.\Psi\right|_{U} ^{n}\left(\eta_{a}\right)}{\rho_{+}^{n}}=\lim _{n \rightarrow \infty} \frac{\eta_{\left.\Psi^{n}\right|_{U}(a)}^{U}}{\rho_{+}^{n}}
$$

and therefore by linearity and continuity of $\iota_{*}$ and using the fact that $\iota_{*}\left(\eta_{u}^{U}\right)=\eta_{u}^{F_{N}}$ for $u \in U, u \neq 1$, we have:

$$
\nu_{+}=\iota_{*}\left(\mu_{+}^{U}\right)=\lim _{n \rightarrow \infty} \frac{\eta_{\Psi^{n}(a)}^{F_{N}}}{\rho_{+}^{n}} .
$$


Using the fact that $\left.\Psi\right|_{U}\left(\mu_{+}^{U}\right)=\rho_{+} \mu_{+}^{U}$, we conclude:

$$
\begin{gathered}
\Psi \nu_{+}=\lim _{n \rightarrow \infty} \Psi \frac{\eta_{\Psi^{n}(a)}^{F_{N}}}{\rho_{+}^{n}}=\lim _{n \rightarrow \infty} \frac{\eta_{\Psi^{n+1}(a)}^{F_{N}}}{\rho_{+}^{n}}= \\
\lim _{n \rightarrow \infty} \frac{\iota_{*}\left(\eta_{\left.\Psi\right|_{U} ^{U}(a)}^{U+1}\right)}{\rho_{+}^{n}}=\iota_{*}\left(\lim _{n \rightarrow \infty} \frac{\left.\eta_{\left.\Psi\right|_{U} ^{n+1}(a)}^{U}\right)=\iota_{*}\left(\left.\Psi\right|_{U}\left(\mu_{+}^{U}\right)\right)=}{\rho_{+}^{n}}\right. \\
\iota_{*}\left(\rho_{+} \mu_{+}^{U}\right)=\rho_{+} \nu_{+} .
\end{gathered}
$$

A similar argument shows that $\Psi^{-1} \nu_{-}=\rho_{-} \nu_{-}$.

Thus $T_{0}, \nu_{+}$and $\nu_{-}$have all the properties required by the conclusion of the proposition.

Theorem 6.2. Let $\varphi_{0}, \psi_{0} \in \operatorname{Out}\left(F_{N}\right)$ be hyperbolic iwips such that the subgroup $\left\langle\varphi_{0}, \psi_{0}\right\rangle \subseteq \operatorname{Out}\left(F_{N}\right)$ is not virtually cyclic. Then there exist $n, m \geq 1$ such that $G=\left\langle\varphi_{0}^{n}, \psi_{0}^{m}\right\rangle \subseteq$ Out $\left(F_{N}\right)$ is free of rank two and every non-trivial element of $G$ is a hyperbolic iwip.

Proof. We already know by the results of Section 4 that if $M_{0}$ is sufficiently big and $n, m \geq M_{0}$ then $G=\left\langle\varphi_{0}^{n}, \psi_{0}^{m}\right\rangle$ is free of rank two, that every nontrivial element of $G$ is atoriodal (i.e. hyperbolic) and that $G$ acts as Schottky group on both, $\overline{\mathrm{CV}}_{N}$ and $\mathbb{P} \operatorname{Curr}\left(F_{N}\right)$ (in the precise sense that the two actions are open 2-generator ping-pong as in Definition 5.3).

Denote $\varphi=\varphi_{0}^{M_{0}}$ and $\psi=\psi_{0}^{M_{0}}$.

We define $G_{1}$ to be the subgroup of $\operatorname{Out}\left(F_{N}\right)$ generated by $\varphi^{M}$ and $\psi^{M}$, for $M \geq 1$ large. Every element $\alpha$ of $G_{1}-\{1\}$ is either conjugate in $G$ to some $\varphi^{k}$ or $\psi^{k}$, with $k \in \mathbb{Z} \backslash\{0\}$, and thus iwip, or else it is conjugate in $G$ to a reduced and cyclically reduced word $u$ in $\varphi, \psi$ which contains the subword $\varphi^{ \pm M}$. After possibly replacing $\alpha$ by $\alpha^{-1}$, we may assume that $u$ contains $\varphi^{M}$ as a subword. Hence $u$ is conjugate in $G$ to a reduced word $u_{1}$ in $\varphi, \psi$ that begins with $\varphi^{m}$ and ends with $\varphi^{n}$ where $m, n \geq \frac{M}{2}-1$ and that represents $\alpha_{1} \in \operatorname{Out}\left(F_{N}\right)$ (which is a conjugate of $\alpha$ ).

Suppose that $\alpha$ is not an iwip, so that $\alpha_{1}$ is not an iwip either. Then by Proposition 6.1 there exist $k \geq 1, T_{0} \in \overline{\mathrm{cv}}_{N}, \nu_{ \pm} \in \operatorname{Curr}\left(F_{N}\right)-\{0\}, \rho_{ \pm}>1$ such that $\left\langle T_{0}, \nu_{+}\right\rangle=\left\langle T_{0}, \nu_{-}\right\rangle=0$ and $\alpha_{1}^{k} \nu_{+}=\rho_{+} \nu_{+}, \alpha_{1}^{k} \nu_{-}=\rho_{-}^{-1} \nu_{-}$. Then $\alpha_{1}^{k}=u_{1}^{k}$ and the word $w=u_{1}^{k}$ in $\varphi, \psi$ still begins with $\varphi^{m}$ and ends with $\varphi^{n}$ and has the form $w=u_{1}^{k}=\varphi^{m} w^{\prime} \varphi^{n}$.

Let $\left[\mu_{ \pm}(\varphi)\right] \in \mathbb{P} \operatorname{Curr}\left(F_{N}\right)$ be the two fixed eigencurrents of $\varphi$ and let $\mu_{ \pm}(\varphi) \in \operatorname{Curr}\left(F_{N}\right)$ be some representatives of them in $\operatorname{Curr}\left(F_{N}\right)$. Let $U_{+}=$ $U_{+}(\varphi)$ and $U_{-}=U_{-}(\varphi)$ be neighborhoods of $\mu_{+}(\varphi)$ and $\mu_{-}(\varphi)$ in $\operatorname{Curr}\left(F_{N}\right)$ provided by Lemma 5.10 .

If $M$ is big enough, then $n, m \geq \frac{M}{2}-1$ are also big enough so that Proposition 5.4 can also be applied to $w$ and $w^{-1}$, and that furthermore, by Lemma 5.9, the forward limit region of $w$ is contained in the image $\mathbb{P} U_{+}$of 
the neighborhood $U_{+}$of $\mu_{+}(\varphi)$, and similarly for the forward limit region of $w^{-1}$ and $U_{-}$.

It follows from Remark 5.2 that for every projectively $w$-fixed current $\mu$ the image $[\mu]$ must be contained in $\mathbb{P} U_{+} \cup \mathbb{P} U_{-}$.

The current $\nu_{+}$is projectively fixed by $\alpha_{1}^{k}=w$ and hence it is contained in the union of the forward and backward limit regions of $w$. Therefore $\left[\nu_{+}\right] \in \mathbb{P} U_{+} \cup \mathbb{P} U_{-}$. Moreover, $\nu_{+}$is projectively fixed by $w$ and is $w$ expanding (since $\rho_{+}>1$ ) while by Proposition 5.4 every projectively fixed current contained in the backward limit region of $w$ is $w$-contracting. Hence $\left[\nu_{+}\right] \in \mathbb{P} U_{+}$so that some non-zero scalar multiple $\nu_{+}^{\prime}$ of $\nu_{+}$satisfies $\nu_{+}^{\prime} \in U_{+}$.

A symmetric argument shows that $\left[\nu_{-}\right] \in \mathbb{P} U_{-}$so that some non-zero scalar multiple $\nu_{-}^{\prime}$ of $\nu_{-}$satisfies $\nu_{-}^{\prime} \in U_{-}$.

Thus Lemma 5.10 applies to $\nu_{+}^{\prime}$ and $\nu_{-}^{\prime}$, so that we obtain $\left\langle T_{0}, \nu_{+}^{\prime}\right\rangle+$ $\left\langle T_{0}, \nu_{-}^{\prime}\right\rangle>0$. But this contradicts the fact that $\left\langle T_{0}, \nu_{+}\right\rangle=\left\langle T_{0}, \nu_{-}\right\rangle=0$.

Hence $\alpha$ is an iwip, as required, which completes the proof of the theorem.

Corollary 6.3. Let $G \subseteq O u t\left(F_{N}\right)$ be a subgroup which contains some hyperbolic iwip, and assume that $G$ is not virtually cyclic. Then $G$ contains a free subgroup of rank two where all non-trivial elements are hyperbolic iwips.

Proof. By Proposition 3.10, $G$ contains two hyperbolic iwips $\varphi$ and $\psi$ some powers of which generate a free group of rank two. Therefore $\langle\varphi, \psi\rangle$ is not virtually cyclic, and the statement of the corollary follows from Theorem 6.2.

\section{REFERENCES}

[1] M. Bestvina, and M. Feighn, A combination theorem for negatively curved groups. J. Differential Geom. 35 (1992), no. 1, 85-101

[2] M. Bestvina and M. Feighn, The topology at infinity of Out $\left(F_{n}\right)$. Invent. Math. 140 (2000), no. 3, 651-692

[3] M. Bestvina, and M. Handel, Train tracks and automorphisms of free groups. Ann. of Math. (2) 135 (1992), no. 1, 1-51

[4] M. Bestvina and M. Feighn, Outer Limits, preprint, 1993; http://andromeda.rutgers.edu/ feighn/papers/outer.pdf

[5] M. Bestvina, M. Feighn, and M. Handel, Laminations, trees, and irreducible automorphisms of free groups. Geom. Funct. Anal. 7 (1997), no. 2, 215-244

[6] M. Bestvina, M. Feighn, and M. Handel, The Tits alternative for $\operatorname{Out}\left(F_{n}\right)$. I. Dynamics of exponentially-growing automorphisms. Ann. of Math. (2) 151 (2000), no. $2,517-623$

[7] M. Bestvina, M. Feighn, and M. Handel, The Tits alternative for $\operatorname{Out}\left(F_{n}\right)$. II. A Kolchin type theorem. Ann. of Math. (2) 161 (2005), no. 1, 1-59

[8] M. Bestvina, M. Feighn, A hyperbolic Out $\left(F_{n}\right)$ complex, preprint, 2008; arXiv:0808.3730

[9] F. Bonahon, Bouts des variétés hyperboliques de dimension 3. Ann. of Math. (2) 124 (1986), no. 1, 71-158

[10] F. Bonahon, The geometry of Teichmüller space via geodesic currents. Invent. Math. 92 (1988), no. 1, 139-162 
[11] M. R. Bridson, and P. de la Harpe, Mapping class groups and outer automorphism groups of free groups are $C^{*}$-simple, J. Funct. Anal. 212 (2004), no. 1, 195-205

[12] P. Brinkmann, Hyperbolic automorphisms of free groups, Geom. Funct. Anal. 10 (2000), no. 5, 1071-1089

[13] Matt Clay and Alexandra Pettet, Twisting out fully irreducible automorphisms, preprint, June 2009, arXiv:0906.4050

[14] M. Cohen and M. Lustig, Very small group actions on R-trees and Dehn twist automorphisms. Topology 34 (1995), no. 3, 575-617

[15] T. Coulbois, A. Hilion, and M. Lustig, $\mathbb{R}$-trees and laminations for free groups $I$ : Algebraic laminations, J. Lond. Math. Soc. (2) 78 (2008), no. 3, 723-736

[16] T. Coulbois, A. Hilion, and M. Lustig, $\mathbb{R}$-trees and laminations for free groups II: The dual lamination of an $\mathbb{R}$-tree, J. Lond. Math. Soc. (2) $\mathbf{7 8}$ (2008), no. 3, $737-754$

[17] T. Coulbois, A. Hilion, and M. Lustig, $\mathbb{R}$-trees and laminations for free groups III: Currents and dual $\mathbb{R}$-tree metrics, J. Lond. Math. Soc. (2) 78 (2008), no. 3, 755-766

[18] M. Culler, K. Vogtmann, Moduli of graphs and automorphisms of free groups. Invent. Math. 84 (1986), no. 1, 91-119

[19] B. Farb and L. Mosher, Convex cocompact subgroups of mapping class groups. Geom. Topol. 6 (2002), 91-152

[20] S. Francaviglia, Geodesic currents and length compactness for automorphisms of free groups, Trans. Amer. Math. Soc. 361 (2009), no. 1, 161-176.

[21] D. Gaboriau, A. Jaeger, G. Levitt, and M. Lustig, An index for counting fixed points of automorphisms of free groups. Duke Math. J. 93 (1998), no. 3, 425-452

[22] D. Gaboriau, and G. Levitt, The rank of actions on $\mathbb{R}$-trees. Ann. Sci. École Norm. Sup. (4) 28 (1995), no. 5, 549-?570

[23] V. Guirardel, Approximations of stable actions on R-trees. Comment. Math. Helv. 73 (1998), no. 1, 89-121

[24] U. Hamenstädt, Word hyperbolic extensions of surface groups, preprint, 2005; arXiv:math.GT/050524

[25] U. Hamenstädt, Subgroups of Out $\left(F_{n}\right)$, a talk at the workshop "Discrete Groups and Geometric Structures", Kortrijk, Belgium, May 2008.

[26] U. Hamenstädt, Lines of minima in Outer space, preprint, November 2009; arXiv:0911.3620

[27] M. Handel and L. Mosher, Subgroup classification in Out $\left(F_{n}\right)$, preprint, August 2009; arXiv:0908.1255

[28] A. Hatcher and K. Vogtmann, The complex of free factors of a free group. Quart. J. Math. Oxford Ser. (2) 49 (1998), no. 196, 459-468

[29] A. Jäger and M. Lustig, Free Group Automorphisms with Many Fixed Points at Infinity, to appear in the Zieschang Gedenkschrift, G\&T monograph series

[30] I. Kapovich, The frequency space of a free group, Internat. J. Alg. Comput. 15 (2005), no. 5-6, 939-969

[31] I. Kapovich, Currents on free groups, Topological and Asymptotic Aspects of Group Theory (R. Grigorchuk, M. Mihalik, M. Sapir and Z. Sunik, Editors), AMS Contemporary Mathematics Series, vol. 394, 2006, pp. 149-176

[32] I. Kapovich, Clusters, currents and Whitehead's algorithm, Experimental Mathematics 16 (2007), no. 1, 67-76

[33] I. Kapovich and M. Lustig, The actions of Out $\left(F_{k}\right)$ on the boundary of outer space and on the space of currents: minimal sets and equivariant incompatibility. Ergodic Theory Dynam. Systems 27 (2007), no. 3, 827-847

[34] I. Kapovich and M. Lustig, Geometric Intersection Number and analogues of the Curve Complex for free groups, Geometry \& Topology 13 (2009), 1805-1833 
[35] I. Kapovich and M. Lustig, Intersection form, laminations and currents on free groups, Geom. Funct. Analysis (GAFA) 19 (2010), no. 5, 1426-1467

[36] I. Kapovich and M. Lustig, Domains of proper dicontinuity on the boundary of Outer space, Illinois J. Math, to appear; arXiv:0902.4263

[37] I. Kapovich and M. Lustig, Stabilizers of $\mathbb{R}$-trees with free isometric actions of $F_{N}$, preprint, 2009; arXiv:0904.1881

[38] I. Kapovich and T. Nagnibeda, The Patterson-Sullivan embedding and minimal volume entropy for Outer space, Geom. Funct. Anal. (GAFA) 17 (2007), no. 4, $1201-1236$

[39] R. P. Kent, and C. J. Leininger, Subgroups of mapping class groups from the geometrical viewpoint. In the tradition of Ahlfors-Bers. IV, 119-141, Contemp. Math., 432, Amer. Math. Soc., Providence, RI, 2007

[40] R. P. Kent, and C. J. Leininger, Shadows of mapping class groups: capturing convex cocompactness, Geom. Funct. Anal. 18 (2008), 1270-1325.

[41] G. Levitt and M. Lustig, Irreducible automorphisms of $F_{n}$ have North-South $d y$ namics on compactified outer space. J. Inst. Math. Jussieu 2 (2003), no. 1, 59-72

[42] M. Lustig, Conjugacy and centralizers for iwip automorphisms of free groups, in "Geometric Group Theory", Trends in Mathematics, 197-224. Birkhäuser Verlag, Basel, 2007

[43] M. Lustig, A generalized intersection form for free groups, preprint 2004

[44] R. Martin, Non-Uniquely Ergodic Foliations of Thin Type, Measured Currents and Automorphisms of Free Groups, PhD Thesis, 1995

[45] H. Masur and Y. Minsky. Geometry of the complex of curves. I. hyperbolicity. Invent. Math., 138 (1999), no. 1, 103-149

[46] L. Mosher, A hyperbolic-by-hyperbolic hyperbolic group. Proc. Amer. Math. Soc. 125 (1997), no. 12, 3447-3455

[47] F. Paulin, The Gromov topology on R-trees. Topology Appl. 32 (1989), no. 3, $197-221$

[48] W. Thurston, Geometry and Topology of 3-manifolds, Lecture Notes, Princeton University, 1977

[49] K. Whittlesey, Normal all pseudo-Anosov subgroups of mapping class groups, Geometry \& Topology 4 (2000), 293-307

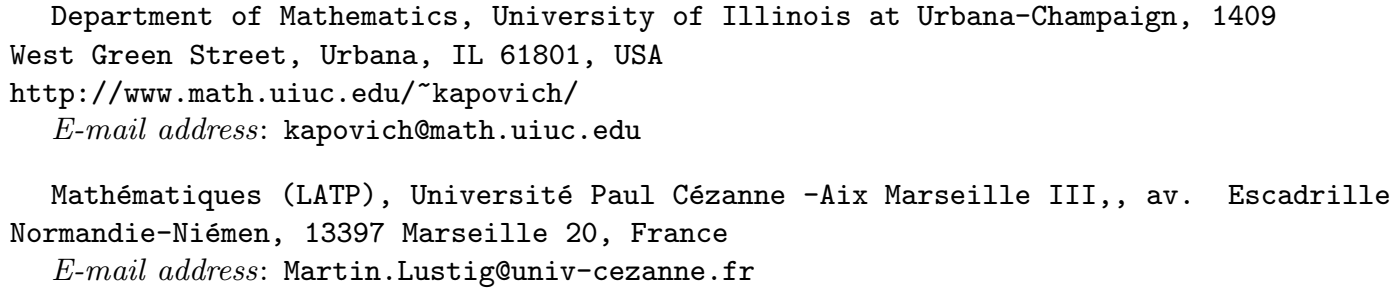

